\title{
The Economic Value of Technical Trading Rules: A Nonparametric Utility-based Approach
}

\author{
Hans Dewachter ${ }^{a, b *}$ and Marco Lyrio ${ }^{a}$ \\ ${ }^{a}$ CES, Catholic University of Leuven \\ ${ }^{b}$ RIFM, Erasmus University Rotterdam
}

March 13, 2002

\begin{abstract}
We adapt Brandt's (1999) nonparametric approach to determine the optimal portfolio choice of a risk averse foreign exchange investor who uses moving average trading signals as the information instrument for investment opportunities. Additionally, we assess the economic value of the estimated optimal trading rules based on the investor's preferences. The approach consists of a conditional generalized method of moments (GMM) applied to the conditional Euler optimality conditions. The method presents two main advantages: (i) it avoids ad hoc specifications of statistical models used to explain return predictability; and (ii) it implicitly incorporates all return moments in the investor's expected utility maximization problem. We apply the procedure to different moving average trading rules for the German mark-U.S. dollar exchange rate for the period 1973-2001. We find that technical trading rules are partially recovered and that the estimated optimal trading rules represent a significant economic value for the investor.
\end{abstract}

Keywords: technical trading rules, exchange rates, nonparametric methods.

J.E.L.: F31, G15.

\footnotetext{
${ }^{*}$ Corresponding author: Hans Dewachter. Center for Economic Studies, Naamsestraat 69, B-3000 Leuven, Belgium. Tel: (+)32 (0)16 326859, Fax: (+)32 (0)16 326796. Email: hans.dewachter@econ.kuleuven.ac.be. We are grateful for financial support from the FWO-Vlaanderen (Project No.: G.0332.01). This paper benefited from discussions with Konstantijn Maes. The latest version of this paper can be downloaded from http://www.econ.kuleuven.ac.be/ew/academic/intecon/Dewachter/default.htm.
} 


\section{Introduction}

Economic theory has been unable to explain in a satisfactory way the apparent success of even the most simple technical trading rules. In general, there seems to be no clear academic consensus regarding neither the profitability nor the source of predictability of these rules. One of the reasons for this relies on the methodology used in this area of research which is still frequently questioned. This methodology can be divided in two steps. The first step involves the determination of a statistical or economic model relating asset returns to a forecasting variable or chartist signal. The second step consists of a statistical analysis of the expected asset return conditional on this signal.

While there is by now some accumulating evidence concerning the profitability of these rules in the foreign exchange market, there is still hardly any consensus regarding the type of statistical or economic structure underlying the observed predictability of such rules. Some studies, for example, argue that this predictability stems from the linear moving average trends in the exchange rates (e.g. Taylor (1980) and LeBaron (1992)). Others claim that nonlinearities are, in fact, the most important source of predictability of chartist rules (e.g. Gençay (1999)). ${ }^{1}$ Given the lack of concensus on the statistical structure, statistical misspecification will obviuously be a major issue in analyzing the results obtained from any specific parametric model for the return generating process.

One possible method to circumvent parametric specifications of the return generating process is to turn to nonparametric alternatives. Brandt (1999) proposes a nonparametric approach that is robust to such model misspecifications. The method adopts a expected utility framework and consists of solving sample analogues of the conditional Euler equations that characterize the portfolio choice of a representative investor. In this paper, we apply Brandt's approach to the foreign exchange market using a simple technical trading signal as the forecasting variable for investment opportunities. We concentrate on this market due to the apparent profitability of chartist rules in this market. Empirical evidence for this can be found in LeBaron (1992, 1999), Gençay (1999), Dewachter (2001) and Taylor (1980), among others. $^{2}$

The use of Brandt's method to analyze technical trading rules provides a number of advantages over the traditional approach mentioned above. First, it answers the main criticism against the standard literature since it avoids ad hoc specifications of statistical or economic models to explain return predictability. The method determines directly the dependence of the optimal portfolio choice on the forecasting variable. In this way, it allows for the most flexible statistical model for the asset return process. Second, the methodology allows the analysis of trading strategies under a consistent economic framework based on the investor's expected utility. In other words, it enables us to give recommendations for an investor with

\footnotetext{
${ }^{1}$ Dewachter (2001) shows that within the framework of a Markov switching model both linear and nonlinear components contribute to the predictability of moving average trading rules. The linear part, however, explains most of the predictability.

${ }^{2}$ LeBaron (2000), however, suggests that previous claims regarding a high performance of technical tranding rules during the 1990's were somewhat exaggerated.
} 
a specific utility function and level of risk aversion. Third, the use of the expected utility framework combined with the nonparametric estimation technique implicitly allows for the dependence of all return moments on the chartist signal. While some studies have, for instance, looked at the Sharpe ratio of trading rules, they are still restrictive since they do not allow for the possible dependence of higher moments (e.g. skewness or kurtosis) on the signal. Fourth, it enables us to give practical portfolio advice based on the investor's optimal trading rule. More specifically, this rule establishes the proportion of an investor's total wealth that should be invested in a certain risky asset according to certain risk preferences. This is in contrast to the simple chartist buy-or-sell recommendation which leaves undetermined the optimal portfolio composition. Chartist rules also make no distinction among different types of investors and their risk preferences. Finally, this method allows us to compute the economic value for the investor of using the estimated optimal trading rule. Only with this final step the investor is able to decide which chartist signal should be used as the forecasting variable for future investment opportunities. In short, in applying Brandt's technique one can provide answers to the question of who and how one should trade upon a given chartist signal. Additionally, it also allows the determination of the economic value of the resulting optimal trading rule. This is achieved without arbitrary model specifications and taking into consideration all possible dependencies of the return moments on the chartist signal.

In the empirical section of the paper we use German mark-U.S. dollar exchange rates for the period 1973-2001. Among the vast number of possible types of technical trading rules, we decide to focus on the moving average type for its simplicity and widespread use. We investigate the case of investors with constant relative risk aversion (CRRA) preferences for different levels of risk aversion. The analysis shows that technical trading rules are partially recovered and, most importantly, that the estimated optimal trading rules represent a significant economic value for investors with reasonable levels of risk aversion.

The procedure presented in this paper can be extended to a multivariate setting with respect to both the number of risky assets composing the portfolio and the number of different signals used as forecasting variables. One can still go one step further. Following Aït-Sahalia and Brandt's (2001) extension of this methodology, one could aggregate these different signals into a single index making use of optimal weighting functions. This would allow investors to determine the most important variables to be tracked and the best way to combine them.

The remainder of the paper is organized as follows. In Section 2, we define the chartist signal used as the forecasting variable, derive the conditional Euler equation applied to the problem, and translate the optimal portfolio choices into certainty equivalent units. Section 3 presents the nonparametric method used to estimate the optimal portfolio composition using standard generalized method of moments (GMM). The result is a simple and easy-to-use estimation technique for optimal "charts-based" trading rules. It also addresses some practical issues related to the implementation of the method. The empirical results are presented in Section 4. Section 5 concludes the paper. 


\section{Optimal Portfolio Choice}

In this section, we start by defining the signal used by the investor as the forecasting variable for investment opportunities. We then derive the first-order condition of the investor's expected utility maximization problem. We end the section with the derivation of the certainty equivalent related to the estimated optimal trading rule.

\subsection{Moving average chartist signal}

Technical, or chartist, analysis tries to identify general patterns in past asset prices without making use of any underlying economic or "fundamental" analysis. The analysis is based on an array of quantitative indicators, or signals, used to derive a variety of different technical trading rules. Despite the diversity of these rules, empirical evidence has shown that even simple rules are able to predict movements in foreign exchange prices, creating successful dynamic trading strategies. The moving average type of rule is one of the most used among these rules and is the one that seems to be robust also in out of sample analysis (see, for instance, Neely et al. (1997)). We use the signal derived from these rules as the forecasting variable observed by the investor. This signal is defined as:

$$
z_{t}=\frac{1}{S} \sum_{j=0}^{S-1} \Delta e_{t-j}-\frac{1}{L} \sum_{j=0}^{L-1} \Delta e_{t-j},
$$

where $e_{t}$ denotes the natural logarithm of the asset price at time $t$, and $S(L)$ denotes the number of observations included in the short (long) window of the moving average signal. $\Delta e_{t}=e_{t}-e_{t-1}$ expresses the asset return at time $t$. This trading signal is supposed to capture the instantaneous momentum in market prices by comparing the average growth rate in the past over a short period with that over a longer period. One usual technical trading rule based on this signal implies a long position in the asset whenever the signal is positive and a short position whenever it is negative.

Irrespective of the statistical quality of the signal $z_{t}$, the chartist trading rule is certainly not designed optimally. ${ }^{3}$ Optimal trading rules use conditional information in order to maximize a certain objective function, which is exactly what is typically missing in chartist trading rules. In the following, we show how this trading signal can be used to derive an optimal trading rule based on the investor's specific utility function.

\subsection{Expected utility framework}

We consider a representative investor with CRRA preferences who allocates a fraction $\alpha$ of wealth $W$ in the risky asset, investing the remaining in a riskless security with return $r_{f}$. The risky asset yields a return of $\widetilde{\Delta} e_{t+1}$ above the risk-free rate. Investment opportunities are time-varying and correlated with the observable forecasting variable $z_{t}$. The investor

\footnotetext{
${ }^{3}$ For instance, it will not be an optimal trading rule whenever agents are risk averse or face constraints on the investment size.
} 
maximizes the one-period ahead expected utility and obtain the following optimal trading rule conditional on the signal observed at time $t$ :

$$
\alpha_{\text {opt }}\left(z_{t}\right)=\arg \max _{\alpha_{t}}\left(E\left[U\left(W_{t+1}\right) \mid z_{t}\right]\right),
$$

with the utility function defined as:

$$
U\left(W_{t}\right)=\left\{\begin{array}{ll}
\frac{W_{t}^{1-\gamma}}{1-\gamma} & \text { for } \gamma>0 \text { and } \gamma \neq 1 \\
\ln \left(W_{t}\right) & \text { for } \gamma=1
\end{array},\right.
$$

where

$$
W_{t+1}=W_{t}\left(1+r_{f}+\alpha_{t} \widetilde{\Delta} e_{t+1}\right)
$$

and where $\gamma$ represents the investor's risk aversion. The optimal trading strategy can then be identified as the portfolio weight $\alpha_{\text {opt }}$ that solves the following conditional Euler equation:

$$
E\left[\frac{\partial U\left(W_{t+1}\right)}{\partial \alpha} \mid z_{t}\right]=0
$$

where the derivative is evaluated at $\alpha_{\text {opt }}\left(z_{t}\right)$. This equation maps the state of nature at each point in time, described by the signal $z_{t}$, into the optimal portfolio choice of the investor.

From the optimality condition in (5), it is easy to show that the design of trading strategies should involve not only the predictability of a chartist signal with respect to the expected asset return but also with respect to the higher moments. For this, we expand the first-order condition in (5) around the expected return:

$$
E\left[\frac{\partial U\left(W_{t+1}\right)}{\partial \alpha} \mid z_{t}\right]=\sum_{k=0}^{\infty} \frac{1}{k !} U_{\alpha}^{(k)} E\left[\left(\widetilde{\Delta} e_{t+1}-E\left(\widetilde{\Delta} e_{t+1} \mid z_{t}\right)\right)^{k} \mid z_{t}\right]=0
$$

where $U_{\alpha}^{(k)}$ denotes the $k$-th derivative of $\partial U\left(W_{t+1}\right) / \partial \alpha$ with respect to $\Delta e_{t+1}$, evaluated in the $z_{t}$-conditional expected wealth. Equation (6) tells us that, unless the agent is risk neutral, the optimal trading rule involves also the predictability of the signal with respect to higher return moments (e.g. conditional variance, skewness or kurtosis). This fact is generally not considered in the standard literature. More importantly, the possibility that all moments enter into the construction of optimal portfolios causes an identification problem in terms of the returns moments to be matched. As a consequence, any statistical model for the return dynamics is forced to incorporate some a priori restrictions on the signal-moment relation. For example, a generalized autoregressive conditional heteroskedasticity (GARCH) type of model would allow for predictability of the signal with respect to the first two return moments but would restrict the higher conditional moments to be constant. For this reason, as suggested by Brandt (1999), in the construction of optimal portfolios, it seems more appropriate to eliminate the intermediate step of positing a statistical model for some of the conditional moments and, instead, use equation (5) directly to derive the optimal portfolio composition. 


\subsection{Certainty equivalent}

The solution of equation (5) characterizes the optimal portfolio choice as a function of the chartist signal. We would also like, however, to determine the economic value for the investor of following this trading strategy. For this, we translate the decision rule expressed by $\alpha_{\text {opt }}$ into a $z_{t}$-conditional certainty equivalent. In other words, we compute the $z_{t}$-conditional certain return $r_{c e}$ that gives the investor the same level of utility as the expected utility derived from a risky investment made according to the optimal trading rule, or:

$$
U\left(W_{c e}\right)=E\left[U\left(W_{t+1} \mid z_{t}=\bar{z}\right)\right] .
$$

where $W_{c e}=W_{t}\left(1+r_{c e}\right)$. Taking a second-order expansion around the initial wealth, $W_{t}$, we have that:

$$
r_{c e}\left(z_{t}=\bar{z}\right)=r_{f}+E\left(\alpha_{o p t} \widetilde{\Delta} e_{t+1} \mid z_{t}=\bar{z}\right)+\frac{1}{2} \frac{U^{\prime \prime} W_{t}}{U^{\prime}} E\left(\left(\alpha_{o p t} \widetilde{\Delta} e_{t+1}\right)^{2} \mid z_{t}=\bar{z}\right)
$$

where the derivatives are evaluated at $W_{t}$. Substituting the derivatives of the utility function gives:

$$
r_{c e}\left(z_{t}=\bar{z}\right)=r_{f}+E\left(\alpha_{o p t} \widetilde{\Delta} e_{t+1} \mid z_{t}=\bar{z}\right)-\frac{1}{2} \gamma E\left(\left(\alpha_{o p t} \widetilde{\Delta} e_{t+1}\right)^{2} \mid z_{t}=\bar{z}\right),
$$

Note that, by construction, this certainty equivalent is downward biased and, therefore, provides a lower bound for the estimated monetary value of the trading rule in consideration. In the following, we show how to use a nonparametric approach to compute this expression.

\section{Nonparametric Approach: Brandt (1999)}

In order to estimate optimal trading rules, Brandt (1999) proposes the use of standard GMM techniques applied to conditional expectations obtained with a nonparametric approach. The method avoids the inconvenient intermediate step of having to specify a statistical model for the asset return. In the following, we present this approach applied to the estimation of the optimality condition in (5). Adapting the method to the above set up, the optimal trading rule $\alpha_{\text {opt }}\left(z_{t}=\bar{z}\right)$ can be estimated using the method of moments:

$$
\alpha_{\text {opt }}\left(z_{t}=\bar{z}\right)=\arg \min _{\alpha_{t}}\left(g Q g^{\prime}\right)
$$

with $Q$ an appropriate weighting matrix and

$$
g=E_{e m p}\left[\frac{\partial U\left(W_{t+1}\right)}{\partial \alpha} \mid z_{t}=\bar{z}\right]
$$

where $E_{e m p}$ represents the nonparametric conditional expectation operator defined on a sample of $T$ observations. The idea is to compute sample analogues to the conditional expectation of $\partial U\left(W_{t+1}\right) / \partial \alpha$. For this, we define the above operator as: 


$$
E_{e m p}\left[X_{t+1} \mid z_{t}=\bar{z}\right] \equiv \frac{\sum_{j=1}^{T} K\left(u_{j}\right) X_{j+1}}{\sum_{j=1}^{T} K\left(u_{j}\right)}
$$

where $K(u)$ denotes a kernel function in $u_{j}, u_{j}=\left(z_{j}-\bar{z}\right) / h$, and where $h$ is the window width or bandwidth of the kernel. This operator gives a weighted average of the observed variable $X_{t}$, where the weight depends on how far each observation is from the reference signal $\bar{z}$.

Results in the literature (see, for instance, Brandt (1999)) show that any reasonable kernel yields asymptotically consistent estimates. Since our problem contains an univariate signal, we are able to adopt a simple normal kernel function, which attributes a greater weight to data points closer to the estimation point:

$$
K\left(u_{j}\right)=\frac{1}{\sqrt{2 \pi}} e^{\left(-\frac{1}{2} u_{j}^{2}\right)} .
$$

The choice of the bandwidth is a more crucial element to the estimation. This parameter scales the difference between the observed $z_{t}$ and the reference signal $\bar{z}$. A large $h$ decreases the variance of the estimator since it increases the number of data points with a higher weight. As a consequence, it increases the potential bias of the estimate since it averages across points that are less similar to the reference point. A smaller $h$ has the opposite effect, it increases the variance and decreases the potential bias. According to standard results in the literature, the bandwidth that guarantees asymptotic consistency ${ }^{4}$ and minimizes the mean squared errors (MSE) of the estimates, balancing the variance against the potential bias, is given by:

$$
h=\lambda \sigma(z) T^{-(1 /(k+4))}
$$

where $\sigma(z)$ is the standard deviation of the variable $z$ and $k$ represents the dimensionality of the signal, equal to one in our case. The proportionality constant $\lambda$ is chosen based on the leave-one-out cross validation technique, which is a data-driven approach, minimizing the MSE in out-of-sample prediction (see Silverman (1986)).

Using the kernel function in (13), the CRRA utility function and the optimal bandwidth selection procedure, Brandt (1999) shows the following asymptotic result for the estimator of the optimal trading rule $\hat{\alpha}$ :

$$
\sqrt{T h}(\hat{\alpha}-\alpha) \longrightarrow N\left(0, \sigma_{\alpha}^{2}\right)
$$

with

$$
\sigma_{\alpha}^{2}=\frac{D(z)^{-1} V(z) D(z)^{-1}}{f(z)} \int_{R} K(u)^{2} d u
$$

where $f(z)$ denotes the nonparametric unconditional density of $z$,

\footnotetext{
${ }^{4}$ Asymptotic consistency requires that $h \rightarrow 0$ fast enough as $T \rightarrow \infty$.
} 


$$
D(z)=\frac{\partial E_{e m p}\left[\frac{\partial U\left(W_{t+1}\right)}{\partial \alpha} \mid z_{t}\right]}{\partial \alpha}
$$

and

$$
V(z)=E_{e m p}\left[\left(\frac{\partial U\left(W_{t+1}\right)}{\partial \alpha}\right)^{2} \mid z_{t}\right] .
$$

The method of Brandt (1999) thus allows for a direct estimation of the optimal portfolio composition, i.e. the weight $\alpha_{\text {opt }}\left(z_{t}=\bar{z}\right)$ conditional on the observation of a certain market signal $z_{t}$. This optimal portfolio composition implicitly defines the optimal (signalconditioned) trading rule. That is, the trading rule implicit in this procedure is given by the mapping from the range of possible values of the signal to the optimal trading action $\alpha$. Using this procedure on the chartist (moving average) information variable $z_{t}$ thus results in the optimal "chart-based" trading rule. This optimal rule can then be used to (i) analyze the information value of the moving average signal by testing whether a rational investor would use this signal in his optimal portfolio decisions, and (ii) to analyze the optimality of the standard trading practices based on the moving average signal, i.e. go long when $z>0$ and short whenever $z<0$ with trading positions that are invariant to the size of the signal. The first issue can be easily tested by looking for $\alpha^{\prime} s$ statistically different from zero, while for the second one should look for constant optimal trading positions across the negative and positive support of $z$. These empirical issues are analyzed in the next section.

\section{Empirical results}

We use daily German mark-U.S. dollar (DM-US\$) spot exchange rates obtained from Datastream for the sample period January 2, 1973 to November 6, 2001, a total of 7526 observations. In the analysis, we disregard the interest rate differential between the countries. ${ }^{5}$ Besides the fact that these data are not readily available, LeBaron (1999) shows that the exclusion of the interest rate differential is unimportant for trading rule results. We also adopt an arbitrary value for the risk free interest rate equal to zero.

We consider the case of a CRRA German investor who uses a daily unidimensional signal $z_{t}$ as the forecasting variable for possible investments in dollars, the only available risky asset, of a fraction $\alpha$ of his total wealth. The analysis is made for three types of signals depending on the number of days incorporated in the short $(S)$ and the long $(L)$ window of the moving average rule in (1). The following signals are used. Signal 1: $S_{d}=10$ and $L_{d}=50$; Signal 2: $S_{d}=20$ and $L_{d}=100$; and Signal 3: $S_{d}=40$ and $L_{d}=200$, where the subscript $d$ indicates the investor's investment horizon (days). The optimal trading rules are derived for three levels of relative risk aversion $(\gamma=5,10$, and 20). The constant $\lambda$ in (14) is chosen,

\footnotetext{
${ }^{5}$ The expression $\widetilde{\Delta} e_{t+1}=\Delta e_{t+1}+r_{f}^{*}-r_{f}$, where $r_{f}^{*}$ represents the risk-free return in the foreign country, can then be simplified to $\widetilde{\Delta} e_{t+1} \cong \Delta e_{t+1}$.
} 
for each type of rule, through a MSE minimizing grid search using the leave-one-out cross validation technique. ${ }^{6}$

In the following, we first present some summary statistics relating the signal $z$ to the moments of the exchange rate returns. We then use the nonparametric approach to compute the expected returns and squared returns as a function of the signal. Up to this point, the analysis is simply a statistical one and intends to investigate the informative content of the signal with respect to the return moments. Subsequently, we use the information contained in the signal to determine the optimal portfolio choice of an investor with a specific utility function and level of risk aversion. We also test for the optimality of a chartist type of trading rule based on the estimated optimal trading rule. We end the empirical analysis for daily returns with the computation of the certainty equivalent for each type of signal and level of risk aversion.

In the last sub-section, we report the results for investment horizons of one week and one month. The weekly and monthly returns are computed as the sum of the previous 5 and 20 daily returns, respectively. We also adopt the same set of signals described above. For the weekly returns, for example, Signal 1 incorporates the previous 2 weeks in its short moving average window and 10 weeks in its long window $\left(S_{w}=2\right.$ and $\left.L_{w}=10\right)$.

\subsection{Summary statistics for moving average trading signals}

Panel A of Table 1 shows the informative content of the daily signal with respect to the sign of the exchange rate return in the following period. In other words, it shows how often an investor would benefit from using a simple chartist rule (buy if $z_{t}>0$ and sell if $z_{t}<0$ ) to direct his investment in dollars. We see that, in our sample, the signals used have no informative content with respect to the direction of daily exchange rate changes. This is in contrast to the results presented in the literature which show for weekly data success rates marginally above 50 percent (e.g. Dewachter (2001)). However, as is shown in the appendix, this lack of predictive ability for changes in exchange rates pertains only to the daily investment horizon. For weekly and monthly investment horizons, we do find the predictive ability of the moving average signal for the direction of exchange rate movements to be significant.

Despite the failure in outperforming the random walk in predicting the direction of exchange rate changes, we show in Panel B of Table 1 that investing according to the chartist rule still results in a significant mean excess return. Irrespective of the rule chosen, investing according to the moving average trading rule implies significant returns of about 3 to 5 percent p.a. upon a positive (long position) signal and of about 4 to 7 percent p.a. upon a negative (short position) signal.

This apparent conflict between sign prediction and mean trading profit returns suggests a dependence of the conditional return moments on the signal. In order to make this link more explicit, we estimate the following regressions, which recover a third-order expansion of the

\footnotetext{
${ }^{6}$ The values used for $\lambda$ for a daily investment horizon are the following. Signal 1: 5.8164; Signal 2: 3.1609 ; and Signal 3: 4.5439.
} 
Table 1: Informative content of chartist signal. Daily investment horizon.

\begin{tabular}{|c|c|c|c|}
\hline & \multicolumn{3}{|c|}{ Type of signal } \\
\hline & Signal 1 & Signal 2 & Signal 3 \\
\hline & $S_{d}=10, L_{d}=50$ & $S_{d}=20, L_{d}=100$ & $S_{d}=40, L_{d}=200$ \\
\hline \multicolumn{4}{|c|}{ Summary statistics } \\
\hline \multicolumn{4}{|c|}{ Panel A: Fraction of correctly predicted signs in exchange rate changes } \\
\hline$z_{t}<0$ & 0.487 & 0.488 & 0.491 \\
\hline$z_{t}>0$ & 0.497 & 0.498 & 0.500 \\
\hline$z_{t}$ & 0.487 & 0.497 & 0.492 \\
\hline \multicolumn{4}{|c|}{ Panel B: Trading profits p.a. terms } \\
\hline$E\left(\Delta e_{t+1} ; z_{t}<0\right)$ & $0.068(0.028)$ & $0.064(0.028)$ & $0.041(0.029)$ \\
\hline$E\left(\Delta e_{t+1} ; z_{t}>0\right)$ & $0.051(0.028)$ & $0.046(0.028)$ & $0.030(0.027)$ \\
\hline$E\left(\Delta e_{t+1} ; z_{t}\right)$ & $0.059(0.019)$ & $0.055(0.019)$ & $0.035(0.019)$ \\
\hline \multicolumn{4}{|c|}{ Regression results } \\
\hline \multirow{2}{*}{\multicolumn{4}{|c|}{$\begin{array}{c}\text { Panel C: Returns } \\
\Delta e_{t+1}=c+\beta_{1} z_{t}+\beta_{2} z_{t}^{2}+\beta_{3} z_{t}^{3}+\varepsilon_{t+1}\end{array}$}} \\
\hline & & & \\
\hline c (x 100) & $-0.005(0.009)$ & $0.002(0.009)$ & $-0.007(0.009)$ \\
\hline$\beta_{1}$ & $0.198(0.055)$ & $0.283(0.083)$ & $0.327(0.113)$ \\
\hline$\beta_{2}$ & $5.353(11.659)$ & $-30.106(23.615)$ & $47.204(47.941)$ \\
\hline$\beta_{3}$ & $-0.709(0.250)$ & $-2.316(0.867)$ & $-6.180(2.410)$ \\
\hline F-test (p-value) & 0.004 & 0.004 & 0.025 \\
\hline \multicolumn{4}{|c|}{ Panel D: Squared returns } \\
\hline \multicolumn{4}{|c|}{$\left(\Delta e_{t+1}\right)^{2}=d+\delta_{1} z_{t}+\delta_{2} z_{t}^{2}+\delta_{3} z_{t}^{3}+v_{t+1}$} \\
\hline $\mathrm{d}(\mathrm{x} 1000)$ & $0.037(0.001)$ & $0.036(0.001)$ & $0.035(0.001)$ \\
\hline$\delta_{1}(\mathrm{x} 10)$ & $0.002(0.008)$ & $0.004(0.012)$ & $-0.014(0.016)$ \\
\hline$\delta_{2}$ & $1.725(0.168)$ & $3.768(0.340)$ & $7.041(0.689)$ \\
\hline$\delta_{3}$ & $-0.001(0.004)$ & $-0.010(0.012)$ & $-0.015(0.035)$ \\
\hline F-test (p-value) & 0.000 & 0.000 & 0.000 \\
\hline
\end{tabular}

Note: Standard errors are in brackets.

conditional return-signal and squared return-signal relation:

$$
\Delta e_{t+1}=c+\beta_{1} z_{t}+\beta_{2} z_{t}^{2}+\beta_{3} z_{t}^{3}+\varepsilon_{t+1}
$$

and

$$
\left(\Delta e_{t+1}\right)^{2}=d+\delta_{1} z_{t}+\delta_{2} z_{t}^{2}+\delta_{3} z_{t}^{3}+v_{t+1} .
$$

The results of these regressions are presented in Panels C and D of Table 1 and in the bottom panels of Figures 1 to 3 (dashed lines). For both regressions, the results point to a statistically significant nonlinear relation between the signal and the returns. For the return regression in (19), one can see in Panel $\mathrm{C}$ of Table 1 that for the three types of signal used both the linear and the cubic terms in the signal are statistically significant. This is reflected in a p-value for the F-test of about 0.003 . The resulting regression lines can be seen in the 
bottom-left panels of Figures 1 to 3 (dashed line). The regression results for the squared returns (eq. 20) are presented in Panel D of Table 1. In this case, we see, as expected, the importance of the squared signal in explaining the squared returns. This gives rise to an approximate symmetric relation between the signal and the squared returns. We find then a significant statistical relation between the chartist moving average signal and the volatility of subsequent exchange rate returns. As far as we know, this aspect of the chartist signal has not been analyzed in the literature.

We conclude from this that the moving average signals used in the analysis contain a significant informational content with respect to both the returns and, surprisingly, the variance of returns in the subsequent period.

\subsection{Nonparametric return moments}

The use of nonparametric techniques allows us to obtain a more flexible representation of the signal-return relationship. Figures 1 to 3 plot the expected return and squared return in function of the chartist signal. The upper panels in these figures show this relation together with the confidence bounds generated using the Künsch (1989) bootstrap method. The lower panels relate the nonparametric mapping to the respective regression lines from (19) and (20). We analyze the results for the return and squared return in turn.

Starting with the mapping for the return, we find evidence of weak predictability of the signal across rules, especially for moderate absolute values of the signal. Furthermore, the mappings implicit in the nonparametric method (solid line, bottom-left panel) differ considerably from the regression line (dashed line), suggesting a strongly nonlinear relation between the signal and the expected return. ${ }^{7}$ This relation also points to some critical values for the signal $z$ beyond which a mean reversion to the long-term trend is predicted, as opposed to a further extrapolation of the short-term trend. This is the case, for instance, of the Signal 1 $\left(S_{d}=10 ; L_{d}=50\right)$ in Figure 1 for values of the chartist signal $z$ larger than $0.8 \%$ in absolute terms. This characteristic is less evident for the Signal $3\left(S_{d}=40 ; L_{d}=200\right)$ presented in Figure 3. In all cases, however, the typical trend extrapolation behavior is observed for intermediate values of $z$ (e.g. between 0 and $0.6 \%$ in absolute terms for the Signal 1, Figure 1 ). Finally, also observe that a value of $z=0$ closely corresponds to the no-change prediction made by practitioners. In general terms, we thus find that the typical trend extrapolation feature of chartist rules is corroborated by the nonparametric regressions as long as we do not observe extreme signals. For the latter sizes, we find a reversal of the short-run trend towards the long-run trend, in contradiction to standard chartist believes.

For the squared returns, we find that the chartist signal also has some potential to explain the heteroskedasticity present in exchange rate returns. More in particular, we find that larger

\footnotetext{
${ }^{7}$ These differences should not come as a surprise. Regression techniques are typically global techniques in that they consider the whole data set in the optimization of the parameters, hence giving a relatively low weight to the tails of the support and simply extending the statistical relations holding for the more dense part of the support. The nonparametric techniques are typically local procedures taking only into account nearby observations.
} 
absolute values of the chartist signal predict an above average expected volatility. Based on the Künsch standard errors, this relation seems to be highly significant. Furthermore, observe that the more inert the rule becomes the less pronounced this relation becomes. Unlike the strong differences between the nonparametric and parametric mappings for the mean, we find similar relations as far as the squared return mapping is concerned.

The above results suggest that the chartist signal $z$ has some predictive content for both the expected return and squared return. In the next section, we show how these mappings enter into the construction of the optimal portfolio composition.

\section{Insert Figures 1 to 3}

\subsection{Optimal trading rules}

Optimal portfolio compositions are obtained directly from equation (5) using Brandt's (1999) nonparametric approach presented in Section 3. We consider investors with three different levels of relative risk aversion parameter $(\gamma=5,10$ and 20). Although there is no consensus on the most appropriate value for this parameter, we believe the ones used cover the range of values reported in the empirical literature. Furthermore, these values are only used to characterize the aggressiveness of the investor, reflected in the estimated optimal trading rule.

Figures 4 to 6 show the optimal trading strategy expressed as the fraction of total wealth invested in U.S. dollars for the different levels of risk aversion. The confidence bounds are computed based on equation (16). A number of observations can be made from these figures. First, the portfolio weights are significantly different from zero for most of the signal values and across the different types of signals, suggesting that trading based on chartist signals may, in fact, improve the investor's expected utility.

\section{Insert Figures 4 to 6}

Second, higher return moments seem to play an important role in the determination of the investment sizes. Comparing Figures 1 to 3 with Figures 4 to 6 one observes that although the direction of investment (going short or long) is mainly determined by the expected return the size of the portfolio also depends on higher moments. More in particular, it can be shown that for larger absolute values of the signals the expected higher variance effectively lowers the absolute size of the investment positions taken. In order to ease visualization, we present a summary of all the portfolio rules in Figure A1 in the appendix.

Third, as expected, the aggressiveness of the trading rule decreases sharply with the increase in the investor's relative risk aversion. In other words, more risk averse agents invest a lower fraction of their wealth in the risky asset (U.S. dollars).

In order to check for the robustness of the optimal trading rules, we also perform the estimation for three sub-periods ${ }^{8}$ within the total sample period. The results are presented

\footnotetext{
${ }^{8}$ The sub-periods chosen are the following. Sub-period 1: from 02/01/1973 to 31/12/1979 (1825 observa-
} 
in appendix B (Figures B1 to B3), which also include the nonparametric results for the expected returns and squared returns. Each row of graphs in these figures correspond to one of the sub-periods in consideration. We compare, for instance, the optimal rules for the entire sample presented in Figure 4 with the ones shown in the three graphs at the right column of Figure B1. Although we observe approximately the same general pattern for the rules in the sub-periods, the optimal trading rules seem, in fact, not to be robust across sub-periods.

\subsection{Trading rule optimality test}

Although Figures 4 to 6 show a clear dependence of the optimal portfolio choice on the signal, the hypothesis of an optimal weight dependent only on the sign and not on the absolute value of the signal should be formally tested. A portfolio choice of the latter kind is similar to the standard chartist rule, although still more sophisticated since it specifies the fraction to be invested in the risky asset depending on the sign of the signal. We refer to it as $\alpha_{\text {tech }}$. The test can be easily done using the GMM techniques presented above. For this, we compute the following test statistics:

$$
J_{N}=\left(\frac{1}{T_{N}} \sum_{z_{t}<0} V\left(z_{t}\right) z_{t}\right) S^{-1}\left(\frac{1}{T_{N}} \sum_{z_{t}<0} V\left(z_{t}\right) z_{t}\right)
$$

and

$$
J_{P}=\left(\frac{1}{T_{p}} \sum_{z_{t}>0} V\left(z_{t}\right) z_{t}\right) S^{-1}\left(\frac{1}{T_{p}} \sum_{z_{t}>0} V\left(z_{t}\right) z_{t}\right)
$$

where

$$
V\left(z_{t}\right)=\frac{\partial U\left(W_{t+1}\right)}{\partial \alpha_{t e c h}}-\left(\frac{\partial U\left(W_{t+1}\right)}{\partial \alpha_{o p t}} ; z_{t}\right)
$$

and where $S$ denotes the variance covariance matrix of $V\left(z_{t}\right) z_{t}$ and $T_{N}$ and $T_{P}$ denote the number of negative and positive signals $z_{t}$, respectively. Under the null hypothesis of optimality of the chartist type of rule, the statistics in equations (21) and (22) are asymptotically distributed as a $\chi^{2}(1)$ random variable. Figures 4 to 6 show the values for the computed $\alpha_{\text {tech }}$ denominated as "chartist". The results of the test are presented in Table 2. As can be inferred from this table, we find strong evidence against the null of an uniform optimal portfolio share, indicating a statistical rejection of the chartist type of moving average rule.

The suboptimality of standard technical trading rules is illustrated in Figures 4 to 6. Figures 4 to 6 show the values for the computed $\alpha_{\text {tech }}$ denominated as "chartist" and represent the optimal portfolio composition conditional on the sign of the moving average signal $z$, as typical chartist trading rules stipulate. As can be seen, the optimal chartist position is the (frequency weighted) average of the optimal trading positions. Although the chartist position tracks the average optimal position (over the negative and positive part of the support of $z$ ), it fails in accounting for the size-dependence of the optimal trading position.

tions); sub-period 2: from 01/01/1980 to 29/12/1989 (2609 observations); and sub-period 3: from 01/01/1990 to $06 / 11 / 2001$ (3092 observations). 
Table 2: Trading rule optimality test (test statistic)

\begin{tabular}{cccc}
\hline \hline & \multicolumn{3}{c}{ Risk aversion $(\gamma)$} \\
\cline { 2 - 4 } Type of signal & 5 & \multicolumn{3}{c}{10} & 20 \\
\hline Signal 1: $S=10 ; L=50$ & & & \\
$z_{t}<0$ & 37.826 & 38.370 & 38.735 \\
$\mathrm{x}$ & $(0.000000)$ & $(0.000000)$ & $(0.000000)$ \\
$z_{t}>0$ & 22.572 & 22.840 & 22.637 \\
& $(0.000002)$ & $(0.000001)$ & $(0.000001)$ \\
Signal 2: $S=20 ; L=100$ & & & \\
$z_{t}<0$ & 14.503 & 14.590 & 11.690 \\
& $(0.000140)$ & $(0.000137)$ & $(0.000628)$ \\
$z_{t}>0$ & 299.983 & 309.482 & 306.281 \\
& $(0.000000)$ & $(0.000000)$ & $(0.000000)$ \\
Signal 3: $S=40 ; L=200$ & & & \\
$z_{t}<0$ & 51.289 & 51.545 & 51.605 \\
& $(0.000000)$ & $(0.000000)$ & $(0.000000)$ \\
$z_{t}>0$ & 316.432 & 333.904 & 353.110 \\
& $(0.000000)$ & $(0.000000)$ & $(0.000000)$ \\
\hline \hline
\end{tabular}

Note: p-values are in brackets.

\subsection{Economic value of trading rules}

To offer the investor a better way of comparison among the optimal trading rules based on different types of signals, we compute the certainty equivalent of each of these rules for the different levels of relative risk aversion. The certainty equivalents translate in economic terms the value for the investor of using these rules. ${ }^{9}$ For the computations, we apply the nonparametric conditional operator defined in (12) to the expression for the certainty equivalent in (9). Results are depicted in Figure 7. Certainty equivalents are stated in per annum terms and are typically of varying size, between $0 \%$ and more than $10 \%$. One can infer easily that for certain ranges of the signal the estimated optimal trading rules, based on chartist signals, produce economically significant gains to standard CRRA utility type of agents. Comparing the certainty equivalents across signals, we see that the Signal $2,\left(S_{d}=20 ; L_{d}=100\right)$, seems to generate on average the larger certainty equivalents.

\section{Insert Figure 7}

\subsection{Investment horizon and transaction costs}

So far we have analyzed optimal trading rules assuming a daily marking to market by fixing the investment horizon to one day. While this analysis showed to result in significant trading positions conditional on the moving average signal, it neglects completely the presence of

\footnotetext{
${ }^{9}$ Obviously, these certainty equivalents are conditional on a certain signal $z_{t}$ and, therefore, do not constitute a proper measure for the certainty equivalent of using the trading rule continuously.
} 
transaction costs and the consequences of opening and closing on a daily basis the speculative positions. It is quite likely that, in practice, the existence of (small) transaction costs decrease or annihilate the profitability of these optimal trading positions. ${ }^{10}$ In order to deal with these issues, we also analyze the optimal trading rules for longer investment horizons, weekly and monthly, effectively reducing the number of market transactions by five and twenty, respectively, and possibly increasing the expected returns over the total investment horizon. We do keep, however, the daily frequency of data thus looking at the optimal portfolio (with investment horizon of one week or one month) that could be formed each day.

In order to save on space, we refer the tables and figures related to the statistical analysis of the signal-return relation to the appendix and only comment on the main issues here. Tables C1 and C3 in the appendix present the summary statistics for each trading signal for investment horizons of one week and one month, respectively. Panel A in both tables show that for weekly and monthly investment horizons, as opposed to daily investment horizon (Table 1 ), all the signals have some informative content with respect to the direction of exchange rate changes. Panel $\mathrm{B}$ shows that investing according to the chartist rules result in significant mean excess returns of about 3 to 6 percent p.a. for a weekly investment horizon and about 2 to 3 percent p.a. for a monthly investment horizon. The nonlinear relation between the signal and the return moments is also present for longer investment horizons (Panels $\mathrm{C}$ and D). As for the weekly investment horizon, in most cases both the linear and the cubic terms in the signal are statistically significant. For two of the signals in the monthly horizon, the quadratic term in the signal is also significant in explaining the mean excess return. Regarding the regression for the squared returns, we also observe the expected importance of the squared signal in explaining the squared returns. For the monthly horizon, both the linear and the cubic terms are also statistically significant.

The crucial aspect about changing the investment horizon is that the signal-return relation may change drastically and hence also the relation between the signal and the optimal trading rule. In order to assess the implications of the change of horizon, we analyze the nonparametric results for each of the signals and holding periods. These results are summarized in Figures 8 and 9 while a more detailed analysis is presented in the Appendix C. Figures 8 and 9 depict the relation between the daily and respectively weekly and monthly expected return and variance for each of the signals. The scatter plots are constructed such that daily returns (variances) are plotted against their weekly or monthly equivalents (conditional on an identical value of the signal $z_{t}$ ). A remarkable feature of these figures is that, in general, the expected returns (variances) tend to be roughly extended linearly over the weekly and monthly investment

\footnotetext{
${ }^{10}$ The level of transaction costs could be measured indirectly using deviations from either the covered interest rate parity or triangular arbitrage conditions, as proposed by Frenkel and Levich (1977). Obviously, such transaction costs are measured per round trip and probably will only apply to major financial players in these markets. Estimates on the round trip costs tend to decrease with the increase in market volumes and liquidity over time. Dooley and Shafer (1986) use a value of about $0.1 \%$. More recent estimates reduce thes costs to about $0.05 \%$ per round trip, e.g. Osler and Chang (1995), Neely et al. (1997). More recent estimates by Al-Awad and Grennes (2002) corroborate the above estimates. Inspection of Figures 1 to 3 clearly show that even small transaction costs as reported above can be an issue when using a daily investment horizon, i.e. expected excess returns often are lower than this $0.05 \%$ benchmark.
} 
horizons. ${ }^{11}$ This can be seen clearly by inspecting the dispersion around the straight line, extending linearly the expected return and variance of daily investment horizons. These extensions thus suggest that the moving average signal is capturing some persistent trend in the exchange rate return and variance, making, for instance, the expected return and variance for the weekly investment horizon around five times as large as the ones for the daily holding period.

Insert Figures 8 and 9

One important implication of the linear extension of mean and variances to longer holding period returns is that the mean-variance relation is constant across investment horizons. To the extent that these moments and, more in particular, their ratio determines the optimal trading position, we do not expect to find substantial differences in the optimal portfolio composition across investment horizons. The optimal portfolio positions for daily and weekly (monthly) holding periods are related in Figure 10 (11). As can be observed, the global picture shows that the optimal portfolio position is relatively independent of the holding period. Figure 10, relating the optimal portfolio positions for daily holding returns to the optimal portfolio positions for weekly holding returns, clearly illustrates the clustering around the 45 degree line for most of the signals. For monthly returns, Figure 11 corroborates this finding, although the observed dispersion is significantly larger in this case. The evidence that the optimal portfolio decomposition is relatively robust with respect to a shift in the investment horizon implies that chartist rules can be used beyond the daily investment period, which in its turn generates less transaction costs as the rebalancing of portfolios is strongly reduced. This reduction in transaction costs possibly allows the application of the above optimal trading rules in practice. More in particular, we find that the certainty equivalents, reported in Figure 7 (for the daily investment horizon), do roughly carry over to the weekly investment horizon (and to a much lesser extent to the monthly holding period). The important difference being that the certainty equivalent for the monthly holding period can be obtained with a much reduced number of trades, decreasing substantially the importance of transaction costs. A detailed analysis along the lines of the one done for the daily investment horizon can be found in Appendix C.

Insert Figures 10 and 11

\section{Conclusion}

In this paper, we use a nonparametric approach to estimate optimal trading rules and to determine their economic value conditioning on a chartist moving average trading signal and

\footnotetext{
${ }^{11}$ Signal 1, i.e the signal with the shortest windows $\left(S_{d}=10\right.$ and $\left.L_{d}=50\right)$ is the exception to this linear extension. This is due to the fact that in the extremes of the signal the linear relation breaks down completely. For more moderate values of the signal, however, the linear relation between daily and weekly (monthly) returns is recovered.
} 
within a standard expected utility framework. The method consists of a GMM approach applied to the first-order condition of the investor's expected utility maximization problem. This approach avoids ad hoc specifications of statistical or economic models to explain return predictability and implicitly incorporates all the return moments in the determination of the optimal portfolio choice.

We apply the methodology to the German mark-U.S. dollar exchange rate for the period from 1973 to 2001. We use different types of moving average trading signals and consider investors with different levels of relative risk aversion. In order to better understand the characteristics of the resulting optimal trading rules, we first analyze the informational content of the selected chartist signals. From nonparametric regressions, we observe clearly evidence of some informational content of the moving average signal. Most interestingly, not only the mean but also the variance of future returns seem to depend on the moving average signal, clearly indicating that the informational content of the chartist signals are not restricted to the expected return.

We subsequently estimate the optimal trading rules conditioning on the chartist signals for CRRA investors with different levels of risk aversion. The optimal trading rules display size dependence and also have a nonlinear relation with respect to the moving average signal. These rules, however, differ significantly from the chartist rules used by practitioners, which stipulate a short (long) position depending on the sign of the signal but independent of its absolute level. We also perform statistical tests for the optimality of a chartist type of rule in which the optimal weight depends only on the sign of the signal. The tests show clearly that this type of rule is sub-optimal for CRRA-type of investors, since they fail to incorporate the observed nonlinear relation between the moving average signal and the first and second return moments. Although we reject the optimality of chartist type of rules, this does not imply that chartist techniques are redundant. On the contrary, we show that using chartist signals to construct optimal trading strategies (as opposed to following chartist trading rules) produces both statistically and economically significant improvements for a representative CRRA investor. Conditional certainty equivalents often surpass the ten percent per annum level.

Finally, we find that the results of the optimal trading rule for a daily investment horizon can in general be extended to longer investment horizons. This finding suggests that the chartist signals pick up some inert stochastic trend in returns (both means and variances). So, even if the daily investment strategy may be practically infeasible due to transaction costs, investing on a weekly horizon effectively reduces these transaction costs without any substantial loss in economic value (certainty equivalent) of the trading rule.

The approach used in this paper can be extended along the lines of Aït-Sahalia and Brandt (2001) to evaluate different types of utility functions, e.g. loss aversion functions, to incorporate multidimensional signals, higher dimensional portfolios or intertemporal optimal trading. These extensions, which are under investigation, were not incorporated in this paper since we intended to focus on the fact that even the simplest chartists signals do present a significant economic value for the investor. 


\section{References}

[1] Aït-Sahalia, Y., and M. Brandt (2001), "Variable Selection for Portfolio Choice", Journal of Finance 56 (4), 1297-1351.

[2] Al-Awad, M. and T.J. Grennes (2002), "Real Interest Rate Parity and Transaction Costs for the Group of 10 Countries", International Review of Economics and Finance 11, 1-10.

[3] Brandt, M. (1999), "Estimating Portfolio and Consumption Choice: A Conditional Euler Equations Approach", Journal of Finance 54 (5), 1609-1645.

[4] Dewachter H. (2001), "Can Markov Switching Models Replicate Chartist Profits in the Foreign Exchange Market", Journal of International Money and Finance 20, 25-41.

[5] Dooley, M.P. and J.R. Shafer (1983), "Analysis of Short-Run Exchange Rate Behavior: March 1973 to November 1981" in Exchange Rate and Trade Instability: Causes, Consequences and Remedies (eds. D. Bigman and T. Taya), Cambridge, MA, Balinger.

[6] Frenkel J.A. and R.M. Levich (1977) "Transaction Costs and Interest Rate Arbitrage: Tranquil versus Turbulent Periods", Journal of Political Economy 85, 1209-1226.

[7] Gençay R. (1999), "Linear, Non-linear and Essential Foreign Exchange Rate Prediction with Simple Technical Trading Rules", Journal of International Economics 47, 91-107.

[8] Künsch, H. (1989), "The Jackknife and the Bootstrap for General Stationary Observations", Annals of Statistics 17, 1217-1241.

[9] LeBaron, B. (1992)," Do Moving Average Trading Rule Results Imply Nonlinearities in Foreign Exchange Markets?", Working Paper, Department of Economics, University of Wisconsin-Madison.

[10] LeBaron, B. (1999), "Technical Trading Rule Profitability and Foreign Exchange Intervention", Journal of International Economics 49, 125-143.

[11] LeBaron, B. (2000), "Technical Trading Profitability in Foreign Exchange Markets in the 1990's", Working Paper, July.

[12] Neely, C., Weller, P. and R. Dittmar (1997), "Is Technical Analysis in the Foreign Exchange Market Profitable? A Genetic Programming Approach", Journal of Financial and Quantitative Analysis 32 (4), 405-426.

[13] Osler, C.L. and P.H.K. Chang (1995) "Head and Shoulders: Not Just a Flaky Pattern" Staff Papers No. 4, Federal Reserve Bank of New York.

[14] Silverman, B. (1986), Density Estimation for Statistics and Data Analysis, London: Chapman and Hall. 
[15] Taylor, S.J. (1980), "Conjectured Models for Trends in Financial Prices, Tests and Forecasts", Journal of the Royal Statistical Society A, 143, 338-362. 

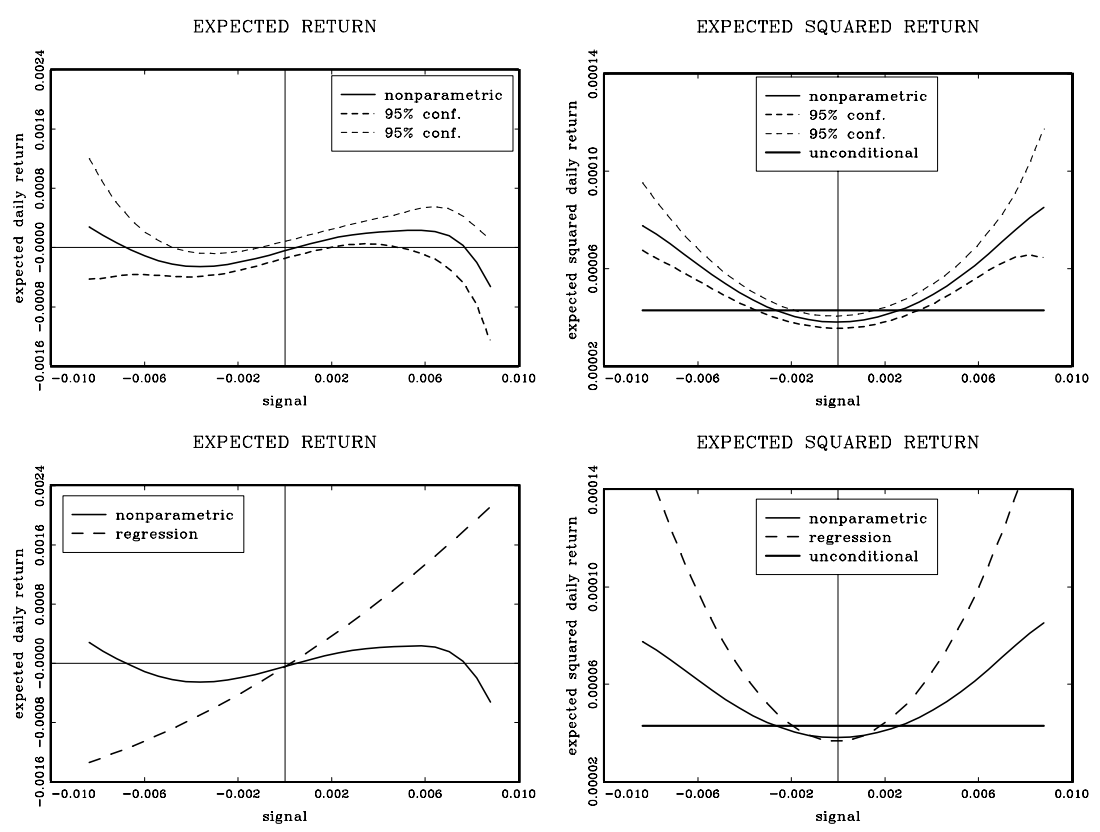

Figure 1: Nonparametric expected daily returns and squared daily returns. Signal 1. 

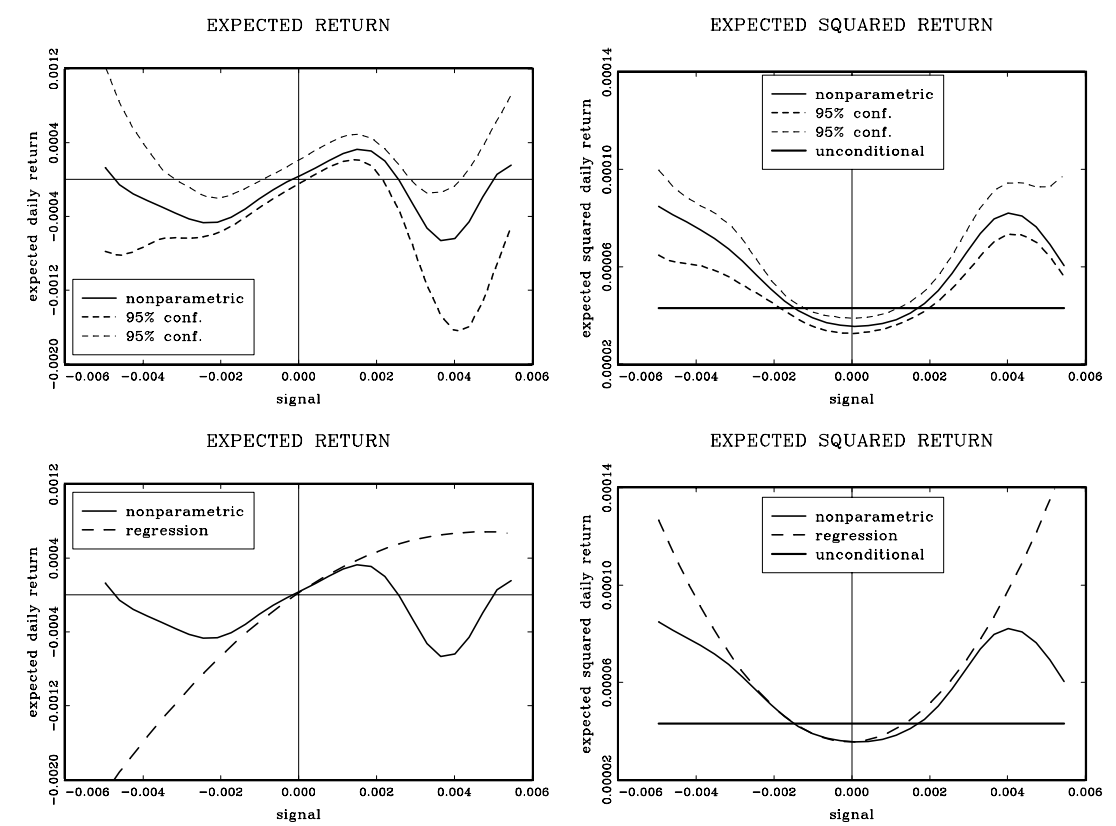

Figure 2: Nonparametric expected daily returns and squared daily returns. Signal 2.
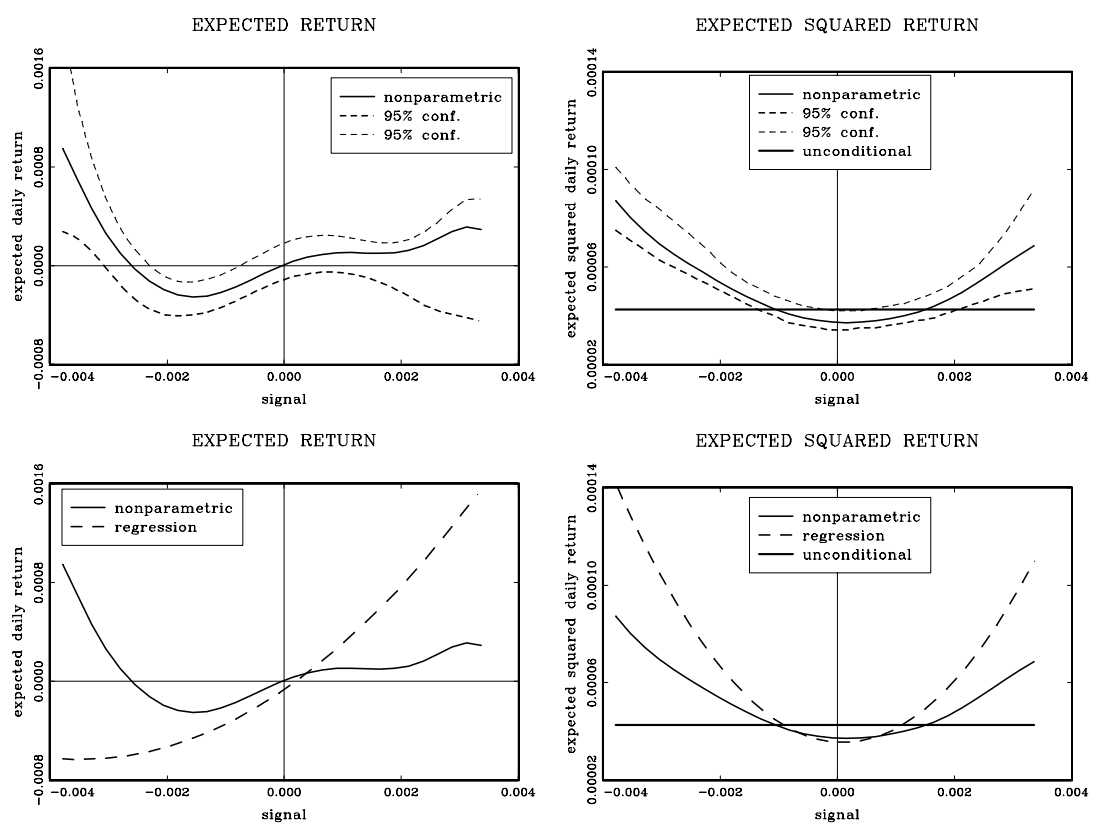

Figure 3: Nonparametric expected daily returns and squared daily returns. Signal 3. 

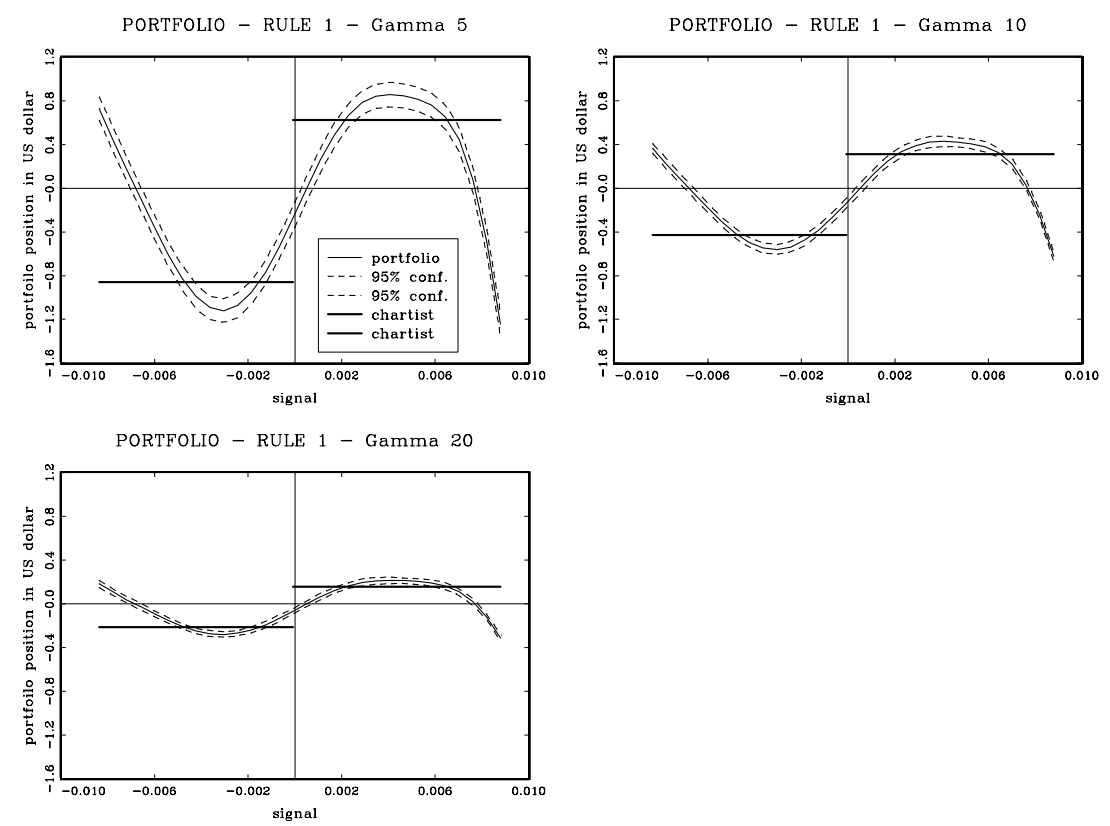

Figure 4: Optimal trading rule. Daily investment horizon. Signal 1.
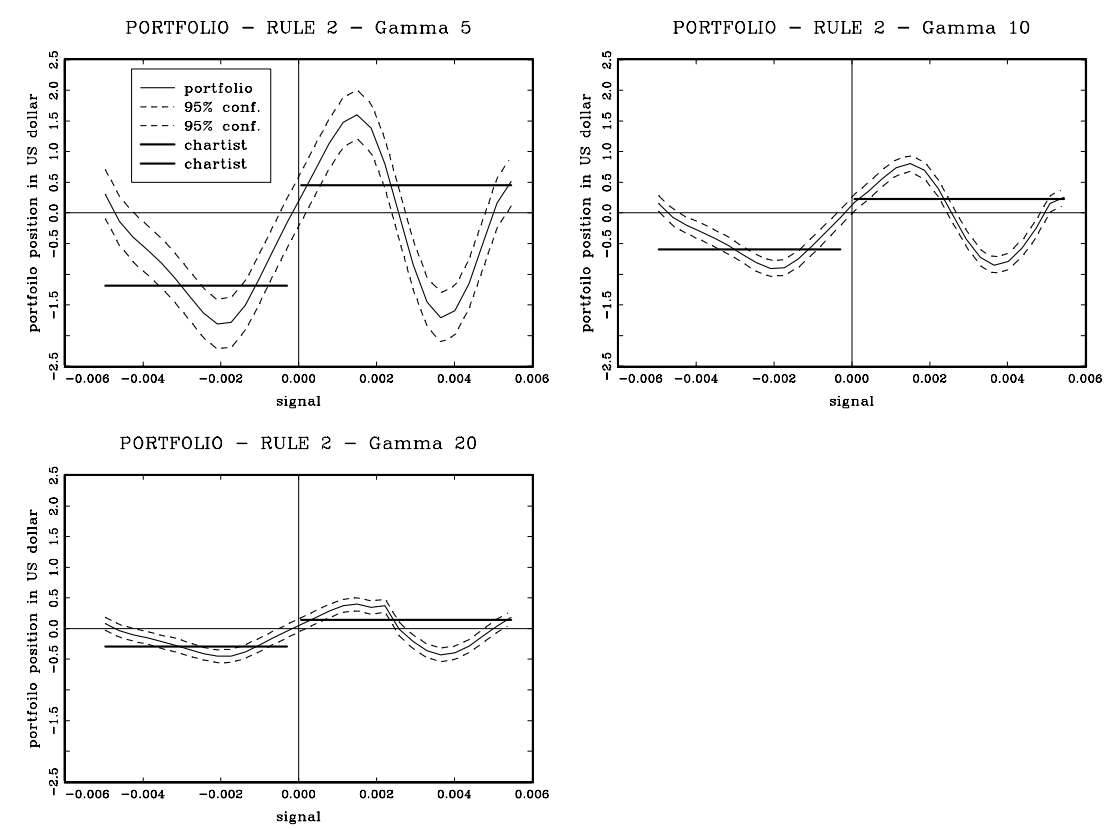

Figure 5: Optimal trading rule. Daily investment horizon. Signal 2. 

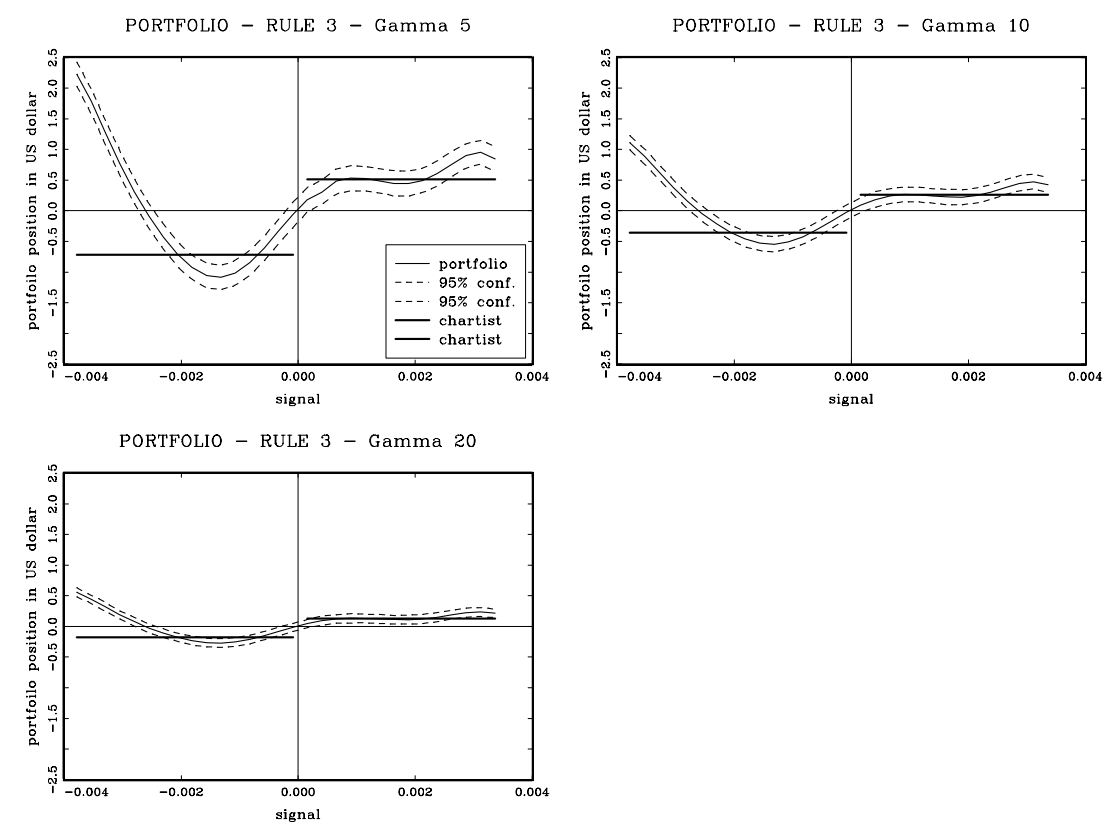

Figure 6: Optimal trading rule. Daily investment horizon. Signal 3.
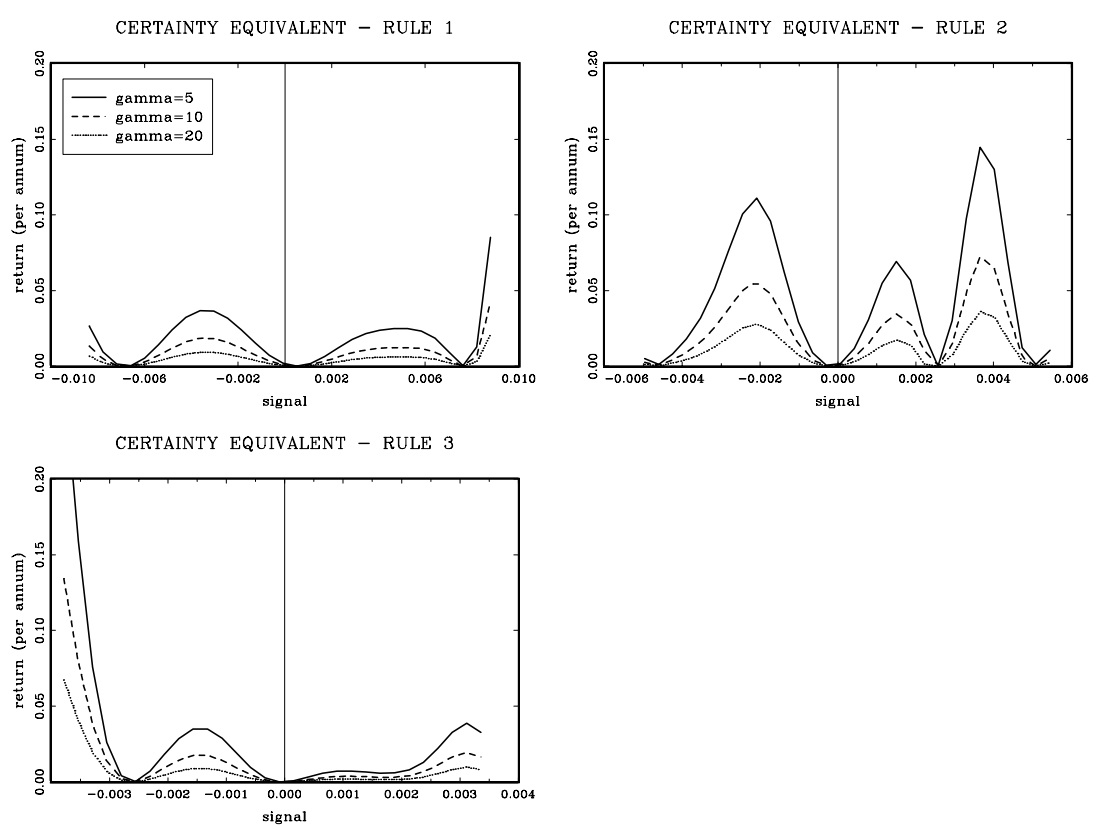

Figure 7: Certainty equivalent. Daily investment horizon. 

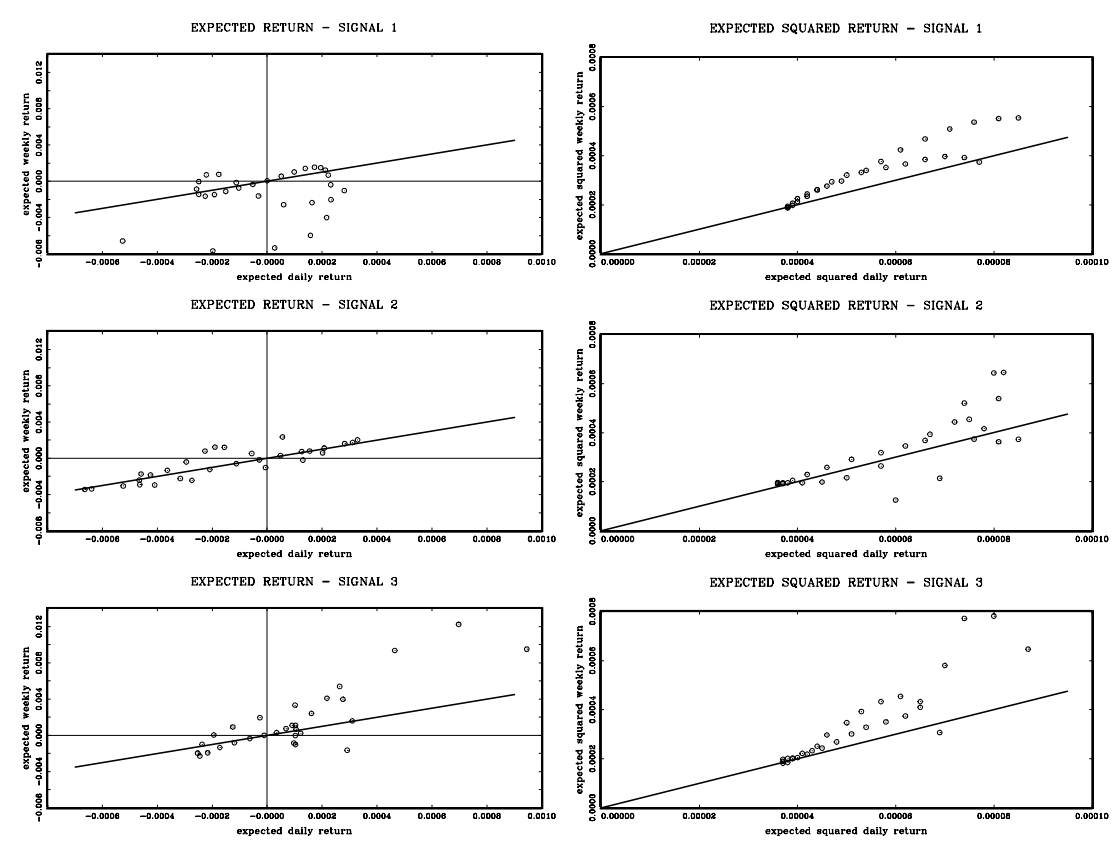

Figure 8: Daily vs. weekly expected returns and expected squared returns.
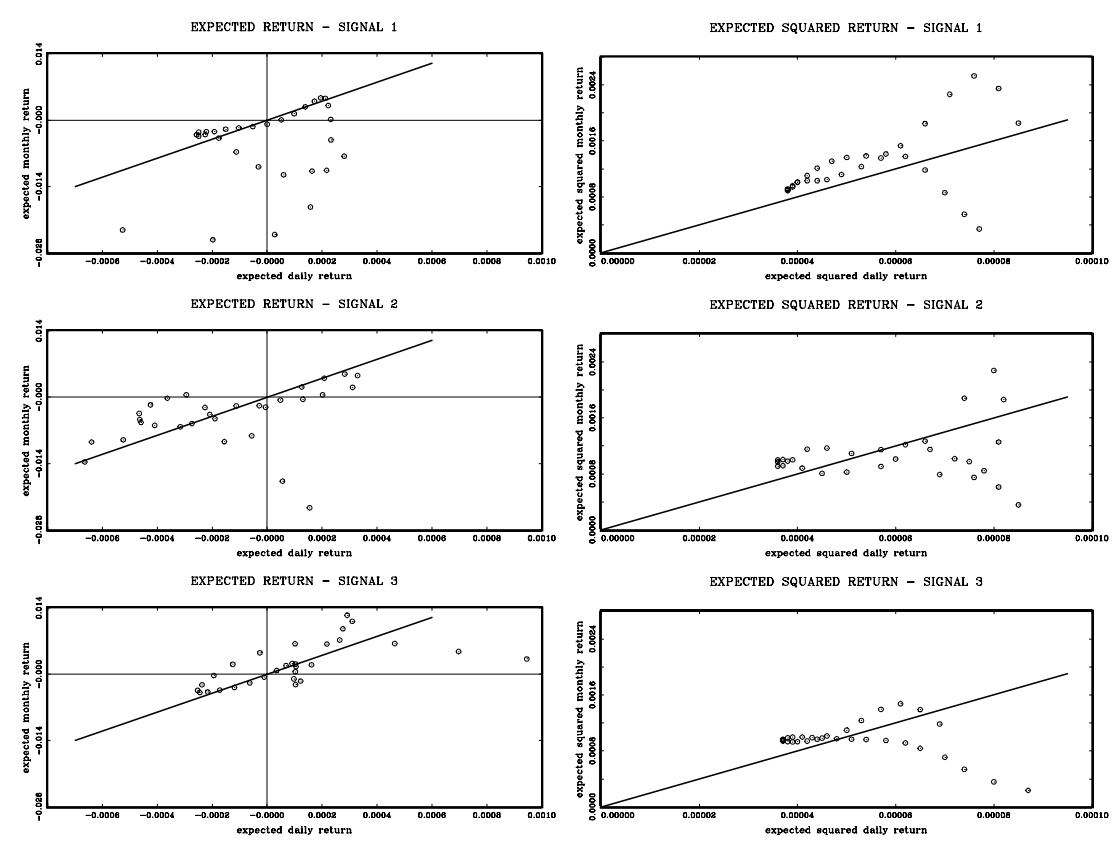

Figure 9: Daily vs. monthly expected returns and expected squared returns. 

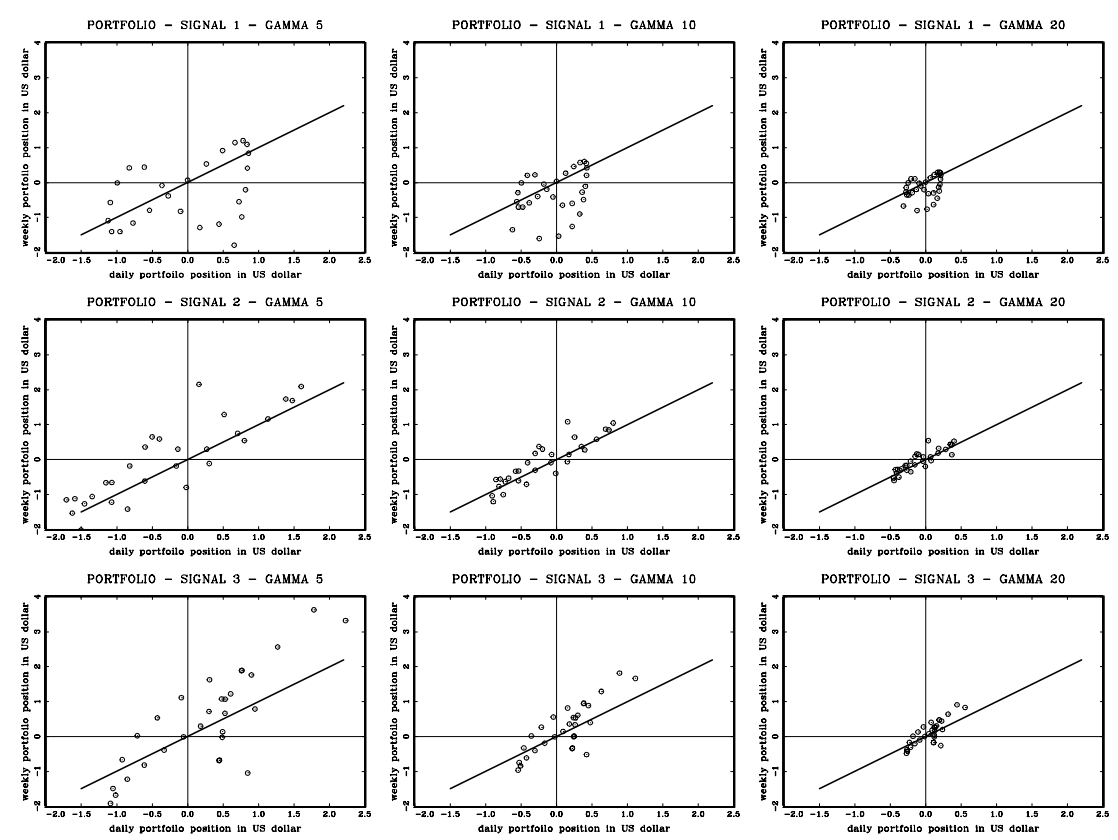

Figure 10: Daily vs. weekly portfolio position in US dollars.
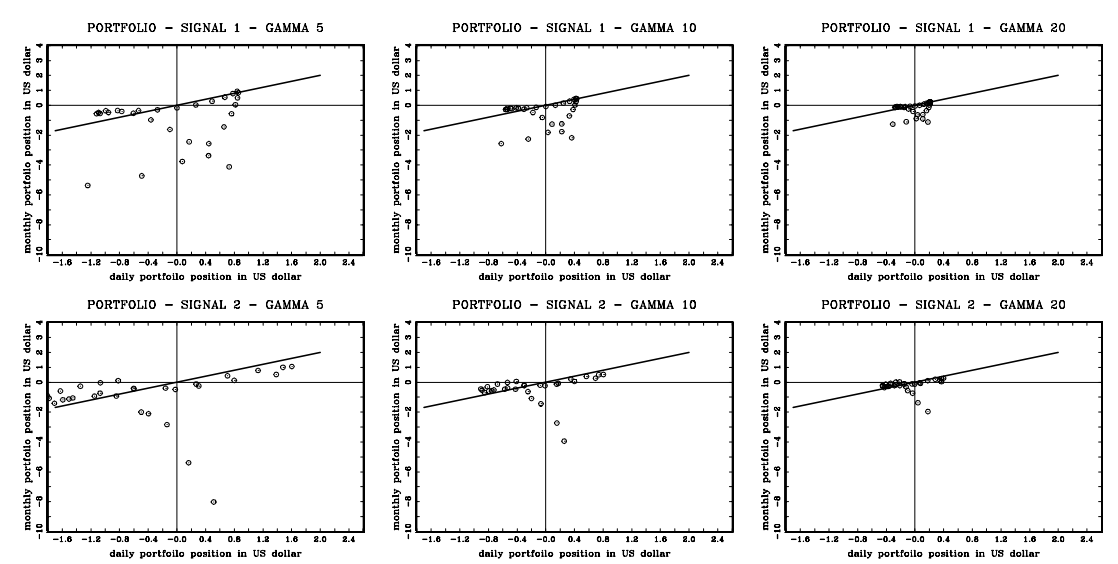

POBTROLIO - SIGNAL. 2 - GAMMA 20

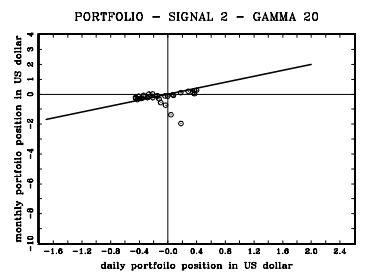

ORTFOLIO - SIGNAL 3 - GAMMA
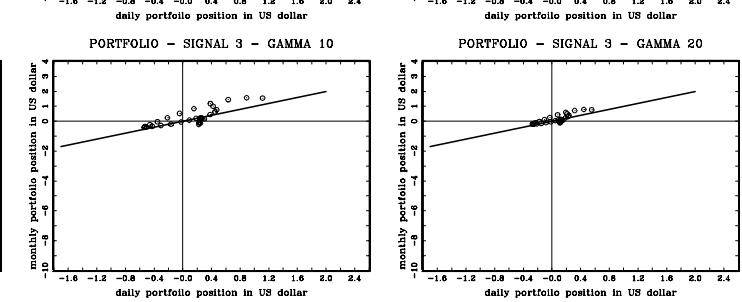

Figure 11: Daily vs. monthly portfolio position in US dollars. 


\section{Appendix}

Appendix A: Summary of portfolio rules for daily investment horizon
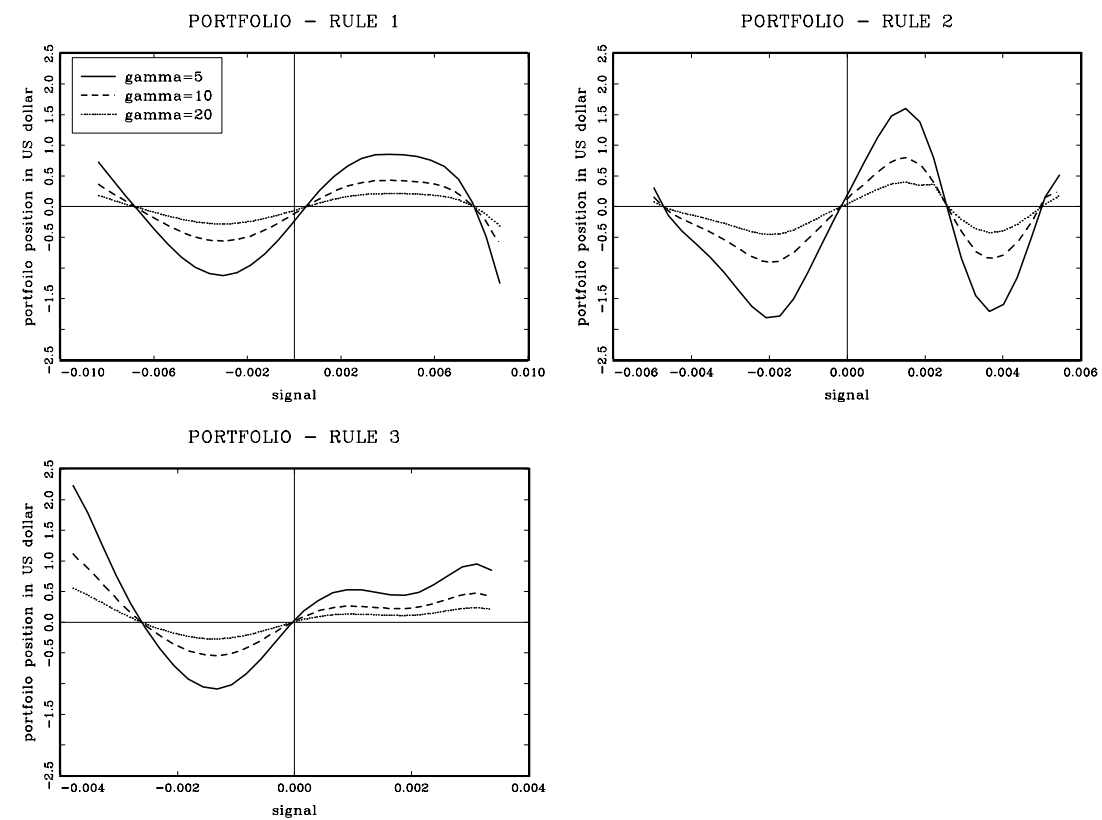

Fig A1: Summary of portfolio rules for daily investment horizon.

\section{Appendix B: Selected results for different sub-periods}
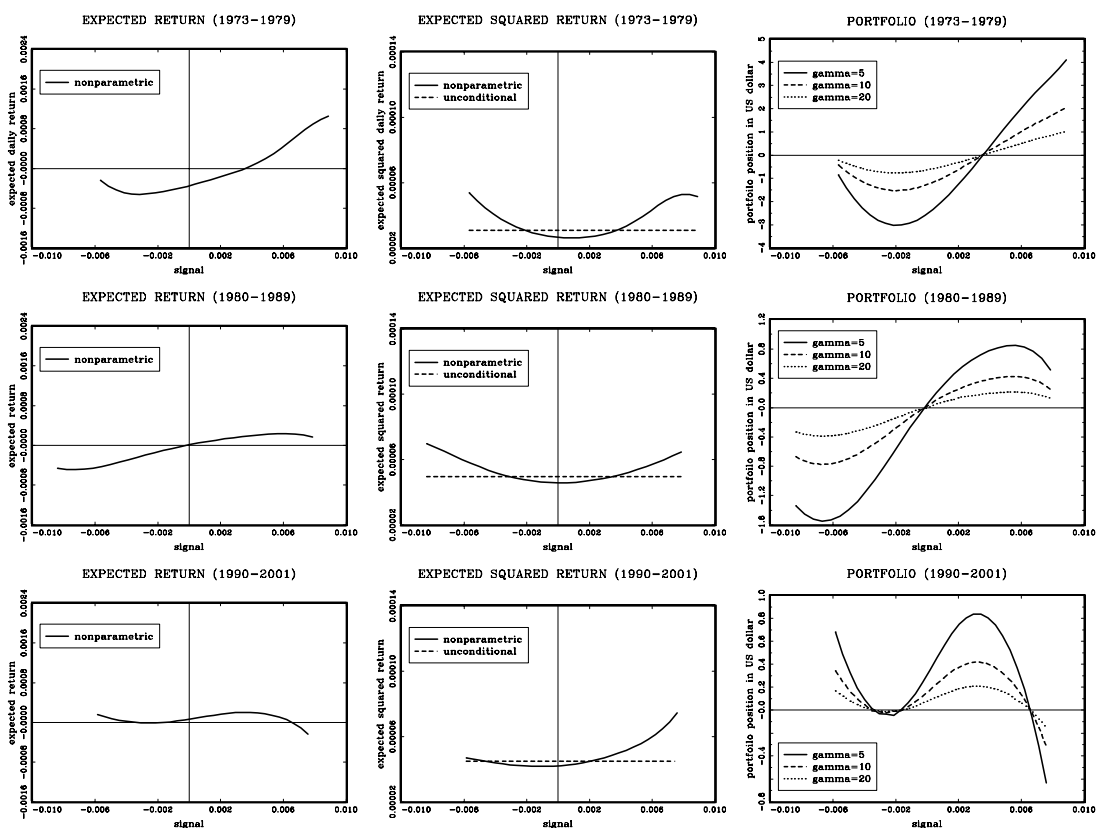

Figure B1: Robustness test. Selected results for Signal 1. Daily investment horizon. 

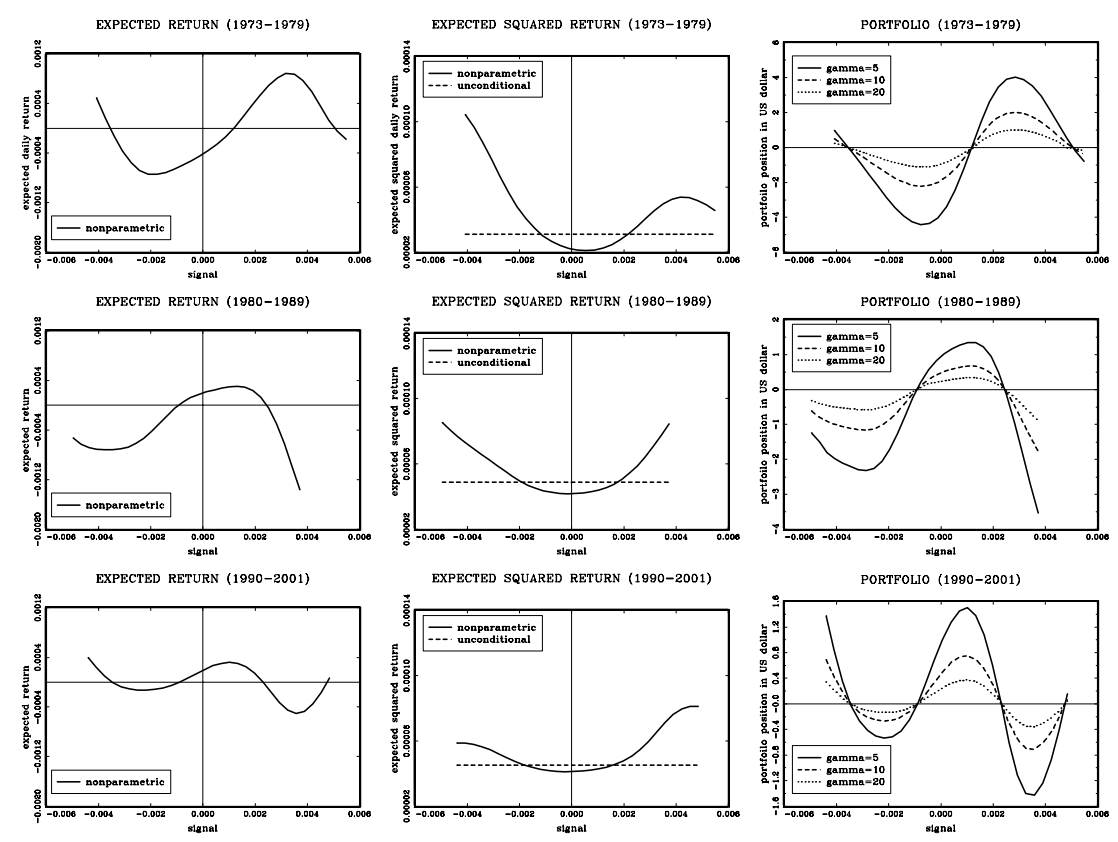

Figure B2: Robustness test. Selected results for Signal 2. Daily investment horizon.
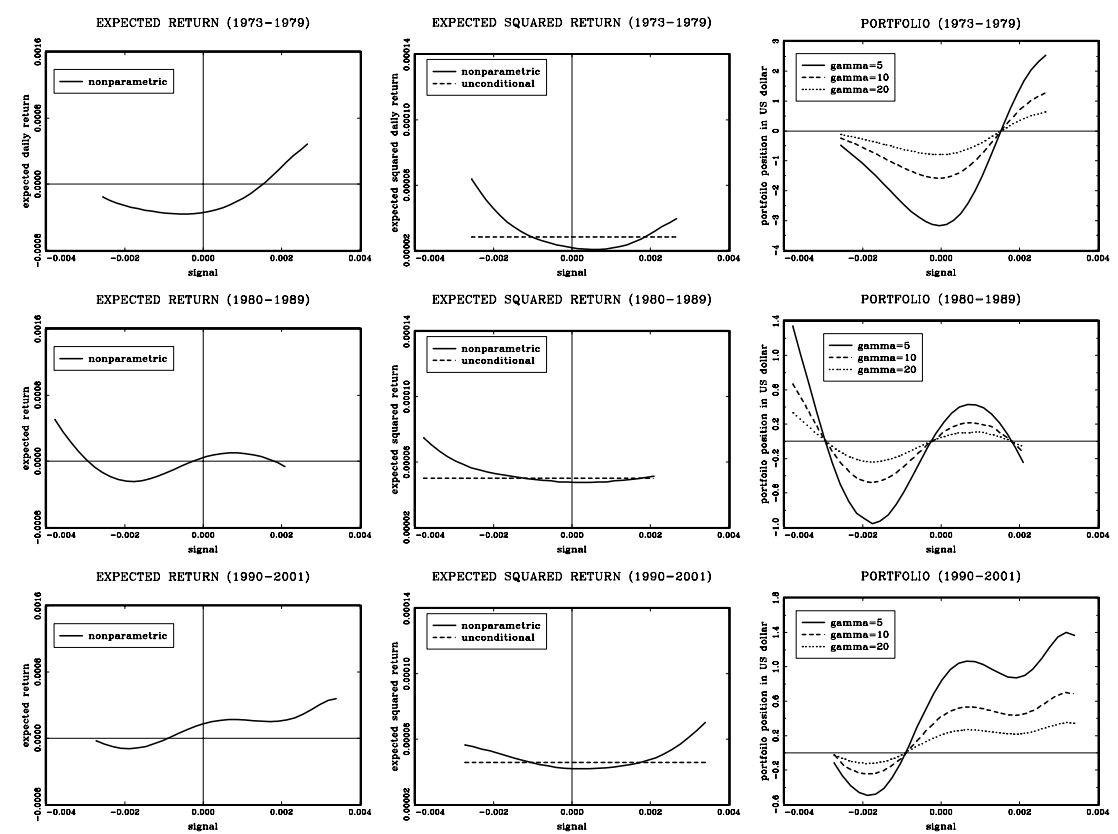

Figure B3: Robustness test. Selected results for Signal 3. Daily investment horizon. 


\section{Appendix C: Selected results for weekly and monthly investment horizons}
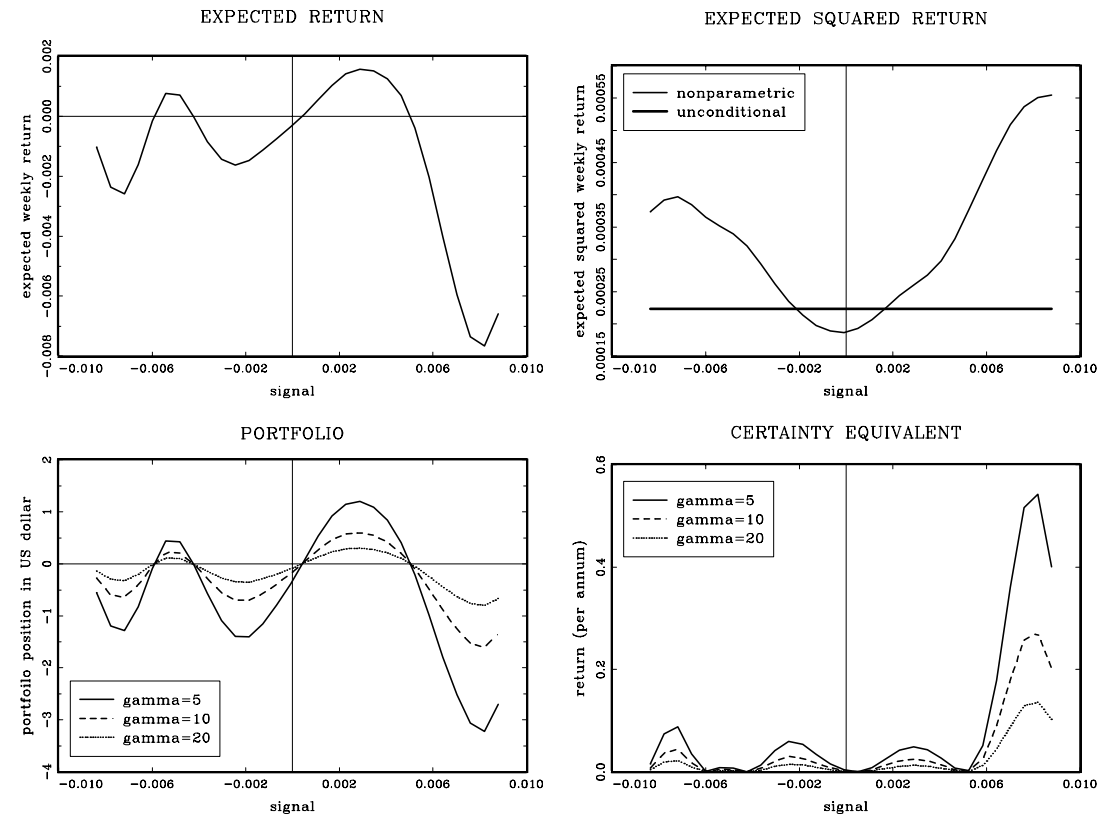

Figure C1: Selected results for weekly investment horizon. Signal 1.
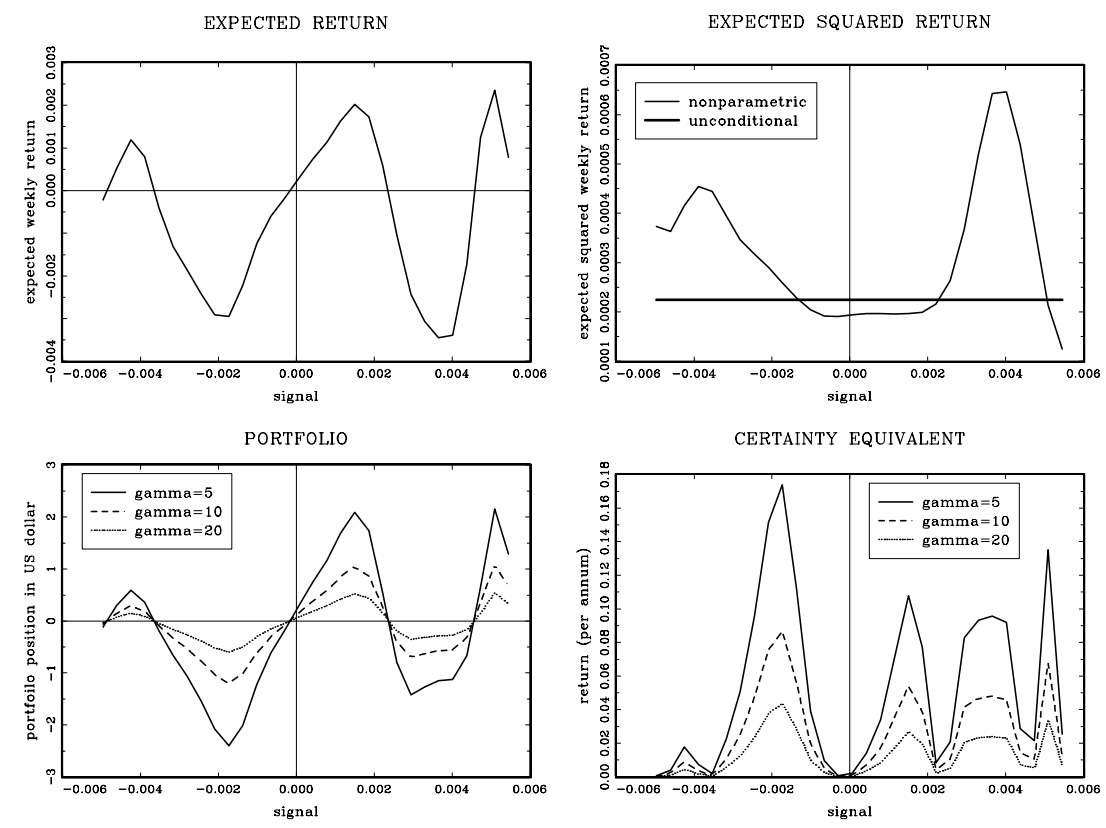

Figure C2: Selected results for weekly investment horizon. Signal 2. 

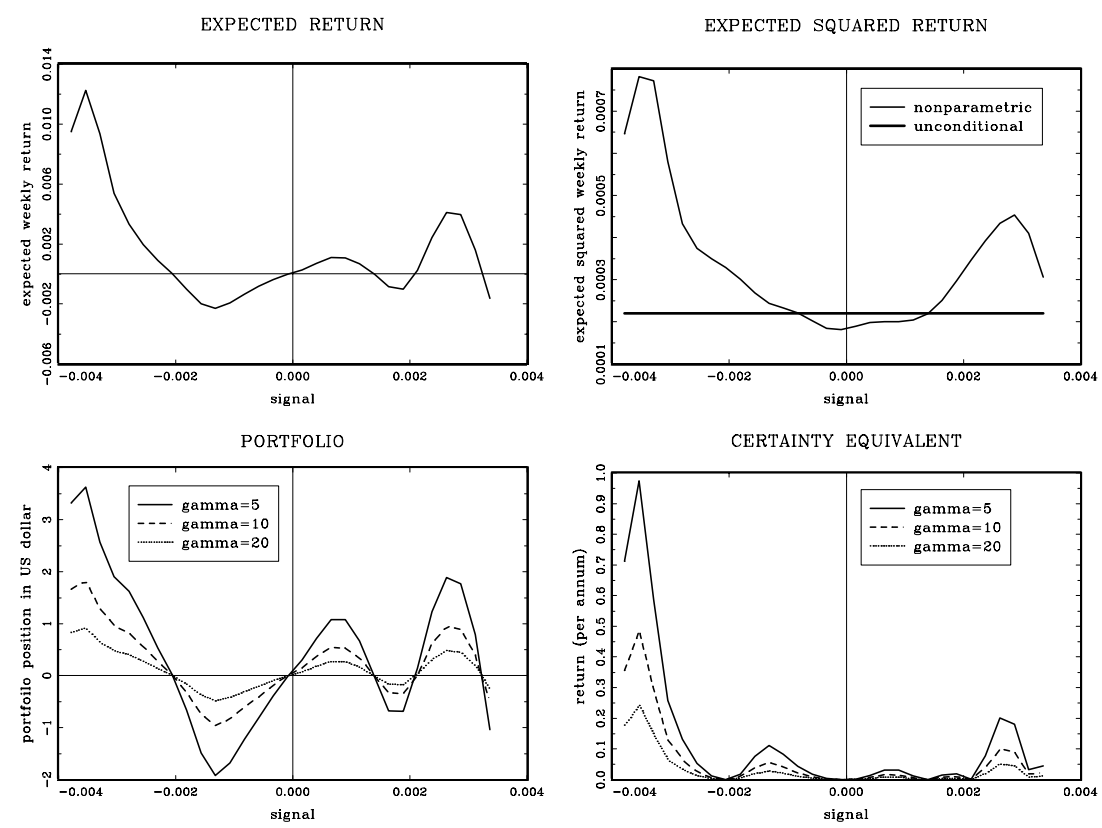

Figure C3: Selected results for weekly investment horizon. Signal 3.
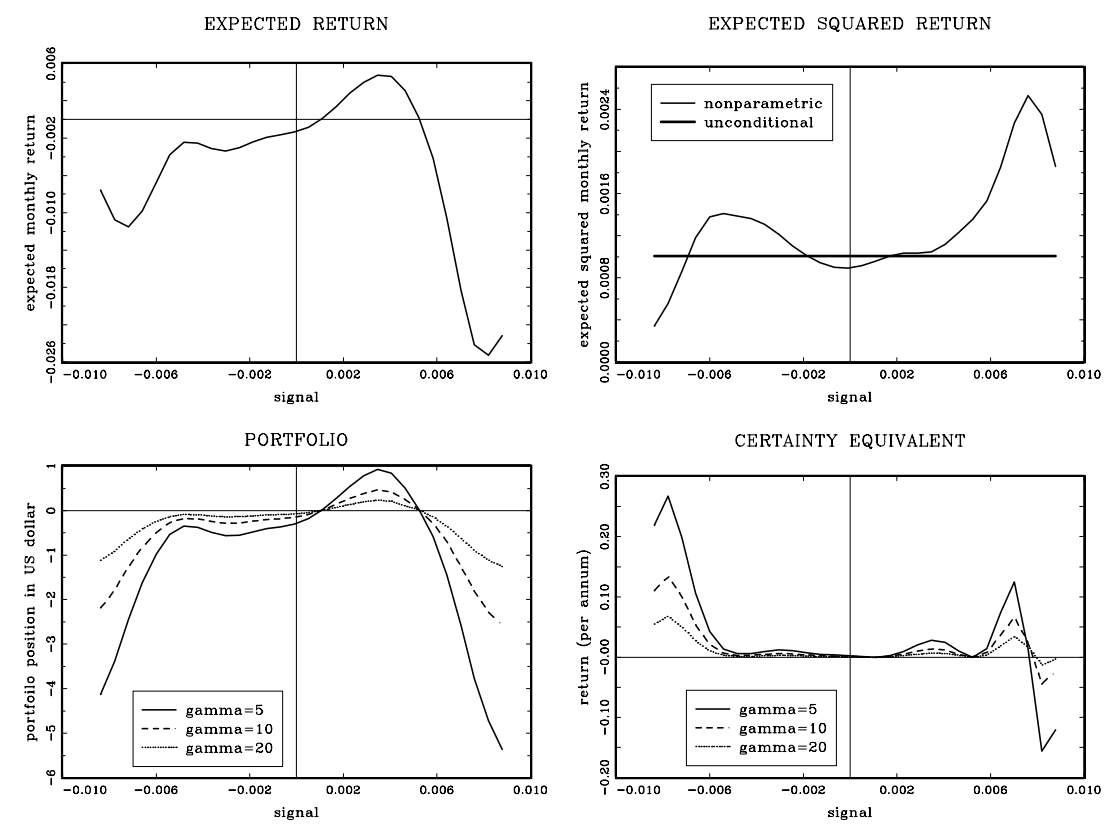

Figure C4: Selected results for monthly investment horizon. Signal 1. 

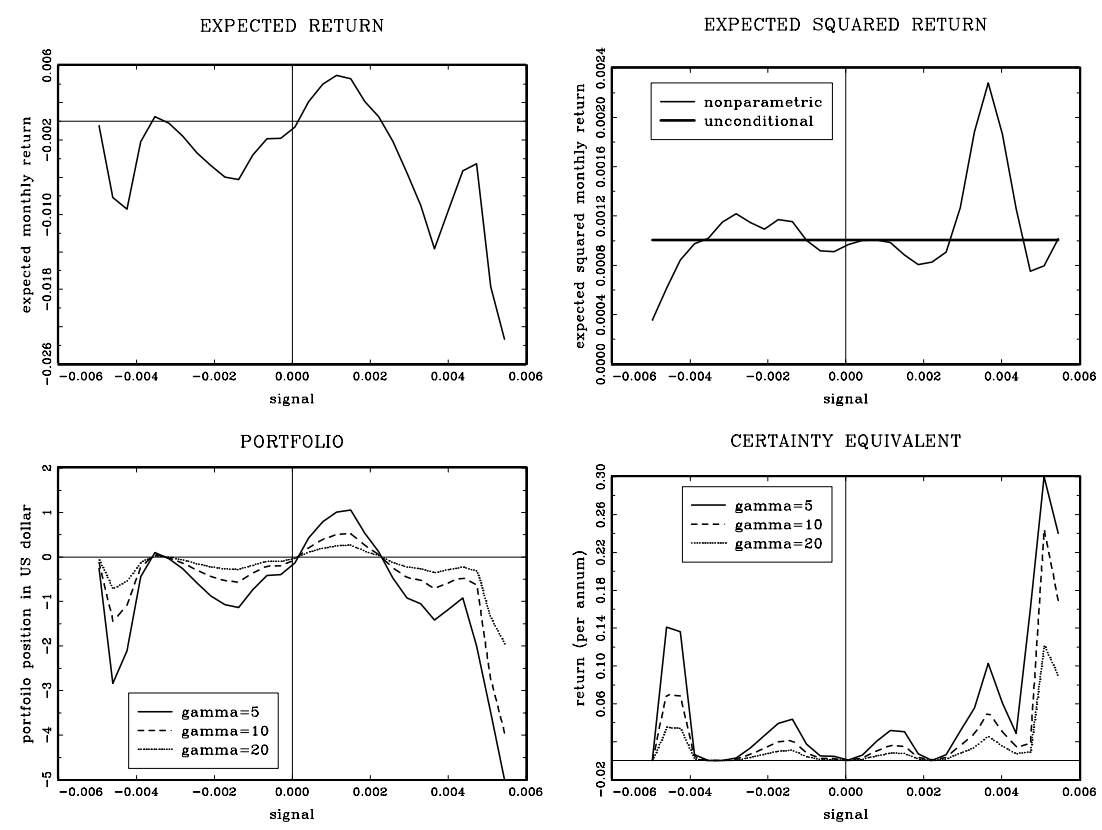

Figure C5: Selected results for monthly investment horizon. Signal 2.
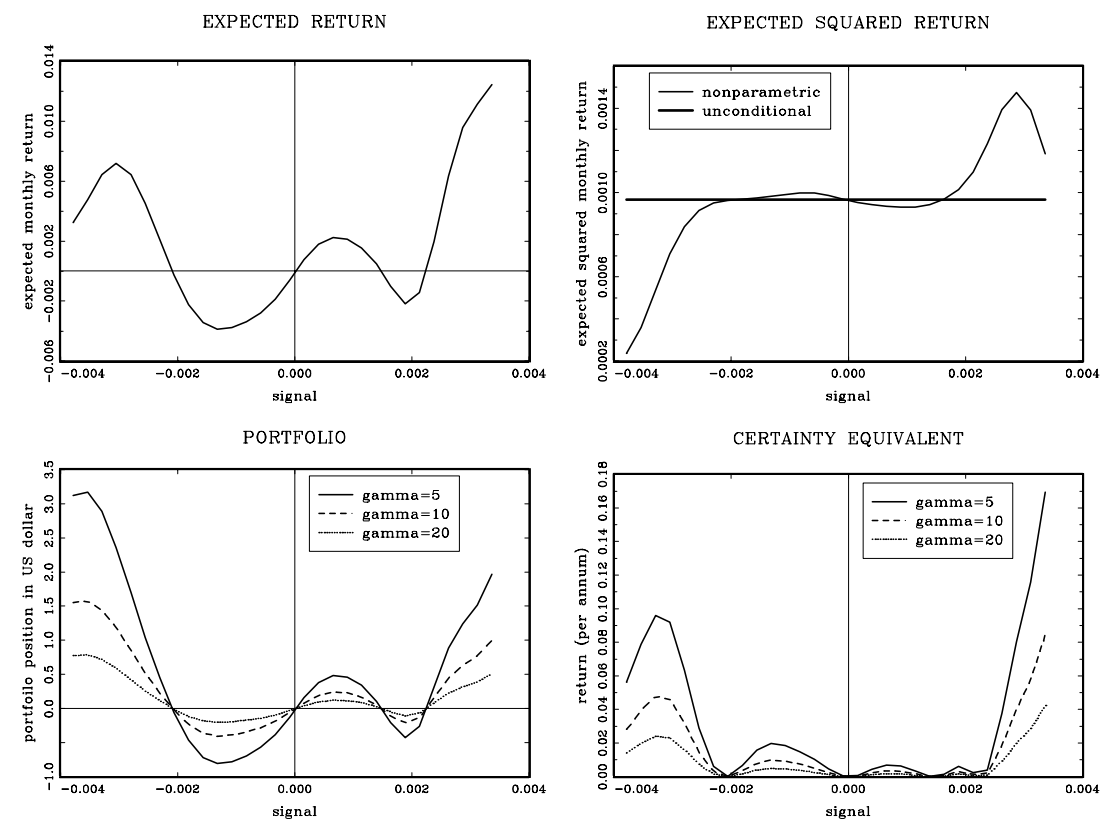

Figure C6: Selected results for monthly investment horizon. Signal 3. 
Table C1: Informative content of chartist signal. Weekly investment horizon.

\begin{tabular}{|c|c|c|c|}
\hline & \multicolumn{3}{|c|}{ Type of signal } \\
\hline & $\begin{array}{c}\text { Signal } 1 \\
S_{w}=2, L_{w}=10 \\
\text { Summary }\end{array}$ & $\begin{array}{l}\quad \text { Signal 2 } \\
S_{w}=4, L_{w}=20 \\
\text { statistics }\end{array}$ & $\begin{array}{c}\text { Signal 3 } \\
S_{w}=8, L_{w}=40\end{array}$ \\
\hline \multicolumn{4}{|c|}{ Panel A: Fraction of correctly predicted signs in exchange rate changes } \\
\hline$z_{t}<0$ & 0.532 & 0.538 & 0.527 \\
\hline$z_{t}>0$ & 0.528 & 0.533 & 0.525 \\
\hline$z_{t}$ & 0.530 & 0.536 & 0.526 \\
\hline \multicolumn{4}{|c|}{ Panel B: Trading profits p.a. terms } \\
\hline$E\left(\Delta e_{t+1} ; z_{t}<0\right)$ & $0.057(0.013)$ & $0.067(0.013)$ & $0.040(0.013)$ \\
\hline$E\left(\Delta e_{t+1} ; z_{t}>0\right)$ & $0.039(0.013)$ & $0.049(0.013)$ & $0.029(0.012)$ \\
\hline$E\left(\Delta e_{t+1} ; z_{t}\right)$ & $0.048(0.009)$ & $0.058(0.009)$ & $0.034(0.008)$ \\
\hline \multicolumn{4}{|c|}{ Regression results } \\
\hline \multicolumn{4}{|c|}{ Panel C: Returns } \\
\hline $\mathrm{c}(\mathrm{x} 100)$ & $-0.148(0.020)$ & $0.007(0.020)$ & $-0.025(0.020)$ \\
\hline$\beta_{1}$ & $0.158(0.025)$ & $0.271(0.037)$ & $0.284(0.051)$ \\
\hline$\beta_{2}$ & $-0.226(1.059)$ & $-5.146(2.134)$ & $6.401(4.288)$ \\
\hline$\beta_{3}$ & $-0.021(0.005)$ & $-0.085(0.016)$ & $-0.196(0.043)$ \\
\hline F-test (p-value) & 0.000 & 0.000 & 0.000 \\
\hline \multicolumn{4}{|c|}{ Panel D: Squared returns } \\
\hline $\mathrm{d}(\mathrm{x} 1000)$ & $0.193(0.006)$ & $0.184(0.006)$ & $0.180(0.006)$ \\
\hline$\delta_{1}(\mathrm{x} 10)$ & $0.004(0.007)$ & $-0.020(0.011)$ & $-0.002(0.015)$ \\
\hline$\delta_{2}$ & $0.316(0.031)$ & $0.772(0.062)$ & $1.394(0.122)$ \\
\hline$\delta_{3}(\mathrm{x} 1000)$ & $0.033(0.132)$ & $0.564(0.456)$ & $-1.845(1.236)$ \\
\hline F-test (p-value) & 0.000 & 0.000 & 0.000 \\
\hline
\end{tabular}

Note: Standard errors in brackets. 
Table C2: Informative content of chartist signal. Monthly investment horizon. Type of signal

\begin{tabular}{|c|c|c|}
\hline $\begin{aligned} & \text { Signal } 1 \\
& S_{m}=0.5, L_{m}=2.5 \\
& \text { Summary s }\end{aligned}$ & $\begin{array}{l}\quad \text { Signal 2 } \\
S_{m}=1, L_{m}=5 \\
\text { tatistics }\end{array}$ & $\begin{array}{c}\text { Signal 3 } \\
S_{m}=2, L_{m}=10\end{array}$ \\
\hline iction of correctly & signs in exchan & 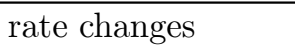 \\
\hline 0.536 & 0.549 & 0.532 \\
\hline 0.531 & 0.544 & 0.529 \\
\hline 0.533 & 0.546 & 0.531 \\
\hline
\end{tabular}

\begin{tabular}{cccc}
$z_{t}$ & 0.533 & 0.546 & 0.531 \\
\hline$E\left(\Delta e_{t+1} ; z_{t}<0\right)$ & $0.032(0.007)$ & $0.042(0.007)$ & $0.036(0.007)$ \\
$E\left(\Delta e_{t+1} ; z_{t}>0\right)$ & $0.014(0.007)$ & $0.027(0.007)$ & $0.025(0.006)$ \\
$E\left(\Delta e_{t+1} ; z_{t}\right)$ & $0.023(0.005)$ & $0.034(0.005)$ & $0.030(0.005)$ \\
\hline
\end{tabular}

\begin{tabular}{|c|c|c|c|}
\hline \multicolumn{4}{|c|}{ Regression results } \\
\hline \multirow{2}{*}{\multicolumn{4}{|c|}{$\begin{array}{c}\text { Panel C: Returns } \\
\Delta e_{t+1}=c+\beta_{1} z_{t}+\beta_{2} z_{t}^{2}+\beta_{3} z_{t}^{3}+\varepsilon_{t+1}\end{array}$}} \\
\hline & & & \\
\hline $\mathrm{c}(\mathrm{x} 100)$ & $-0.042(0.042)$ & $0.020(0.042)$ & $-0.095(0.042)$ \\
\hline$\beta_{1}$ & $0.082(0.013)$ & $0.155(0.020)$ & $0.121(0.027)$ \\
\hline$\beta_{2}$ & $-0.194(0.140)$ & $-1.028(0.281)$ & $1.570(0.561)$ \\
\hline$\beta_{3}(\mathrm{x} 100)$ & $-0.057(0.0150)$ & $-0.337(0.0517)$ & $-0.320(0.142)$ \\
\hline F-test (p-value) & 0.000 & 0.000 & 0.000 \\
\hline \multicolumn{4}{|c|}{ Panel D: Squared returns } \\
\hline \multicolumn{4}{|c|}{$\left(\Delta e_{t+1}\right)^{2}=d+\delta_{1} z_{t}+\delta_{2} z_{t}^{2}+\delta_{3} z_{t}^{3}+v_{t+1}$} \\
\hline $\mathrm{d}(\mathrm{x} 1000)$ & $0.926(0.021)$ & $0.948(0.021)$ & $0.929(0.020)$ \\
\hline$\delta_{1}(\mathrm{x} 10)$ & $-0.015(0.007)$ & $-0.031(0.010)$ & $-0.025(0.013)$ \\
\hline$\delta_{2}$ & $0.048(0.007)$ & $0.051(0.014)$ & $0.043(0.027)$ \\
\hline$\delta_{3}(\mathrm{x} 1000)$ & $0.019(0.007)$ & $0.098(0.026)$ & $0.232(0.068)$ \\
\hline F-test (p-value) & 0.000 & 0.000 & 0.000 \\
\hline
\end{tabular}

Note: Standard errors in brackets. 